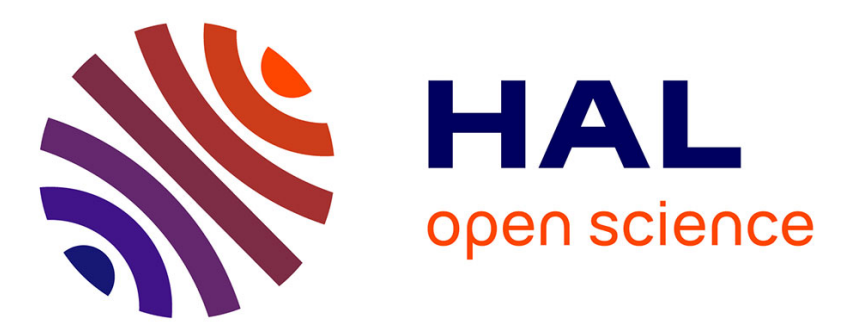

\title{
The Coarse Mesh Condensation Multiscale Method for parallel computation of heterogeneous linear structures without scale separation
}

\author{
Minh Vuong Le, Julien Yvonnet, Nicolas Feld, Fabrice Detrez
}

\section{To cite this version:}

Minh Vuong Le, Julien Yvonnet, Nicolas Feld, Fabrice Detrez. The Coarse Mesh Condensation Multiscale Method for parallel computation of heterogeneous linear structures without scale separation. Computer Methods in Applied Mechanics and Engineering, 2020, 363, pp.112877. hal-02451108

\author{
HAL Id: hal-02451108 \\ https://hal.science/hal-02451108
}

Submitted on 23 Jan 2020

HAL is a multi-disciplinary open access archive for the deposit and dissemination of scientific research documents, whether they are published or not. The documents may come from teaching and research institutions in France or abroad, or from public or private research centers.
L'archive ouverte pluridisciplinaire HAL, est destinée au dépôt et à la diffusion de documents scientifiques de niveau recherche, publiés ou non, émanant des établissements d'enseignement et de recherche français ou étrangers, des laboratoires publics ou privés. 


\title{
The Coarse Mesh Condensation Multiscale Method for parallel computation of heterogeneous linear structures without scale separation
}

\author{
Minh Vuong Le ${ }^{\mathrm{a}, \mathrm{b}}$, Julien Yvonnet ${ }^{\mathrm{a}, *}$, Nicolas Feld $^{\mathrm{b}}$, Fabrice Detrez $^{\mathrm{a}}$ \\ ${ }^{a}$ Laboratoire Modélisation et Simulation Multi Echelle, Université Paris-Est, MSME UMR 8208 CNRS, 5 bd Descartes, 77454 Marne-la-Vallée, \\ France \\ ${ }^{b}$ Safran Tech, Etablissement Paris Saclay, rue des Jeunes Bois-Châteaufort, 78114 Magny-les-Hameaux, France
}

\begin{abstract}
A Coarse Mesh Condensation Multiscale Method (CMCM) is proposed to solve large heterogeneous linear structures without scale separation assumption. The technique aims to approximate the full field solution in heterogeneous structures by performing parallel calculations on subdomains. In the linear case, treated in this paper, direct linear relationships can be established between a reduced number of parameters describing Dirichlet boundary conditions on the subdomains boundaries and the degrees of freedom of a coarse mesh. The problem associated with the coarse mesh can be solved in one iteration and allows reconstructing the fine mesh solution in all subdomains. The accuracy of the method is analyzed through benchmarks involving subdomains crossed by the interfaces. Appplications to large industrial finite element applications are presented, including one involving around 1.3 billion degrees of freedom.
\end{abstract}

Keywords: Multiscale methods, composites, parallel calculations, CMCM

\section{Introduction}

Composite materials constitute a mature technology in many industrial fields including the aircraft and automotive industries. With the constant increase in computer performance, numerical methods play a crucial role in the study of heterogeneous structures, including those made of composites. They allow the analysis of these materials without performing expensive laboratory experiments. In some situations, it may be useful to solve the structural problem with a full description of all heterogeneities. For example when the characteristic size of the heterogeneities is not much smaller than the dimensions of the sample, classical homogenization methods fail to describe the local fields and up to a certain precision even the global response. However, solving even small samples with all heterogeneities in complex materials, like woven composites, is a challenge as the corresponding finite element problem can involve billions of degrees of freedom.

One possible strategy for tackling this issue is parallel computing based on domain decomposition methods. In the past years, many techniques have been proposed, including Finite Element Tearing and Interconnecting (FETI) [1] and Balanced Domain Decomposition (BDD) [2], where the BDD method is referred to as a primal approach and FETI as a dual approach. The above methods are based on the decomposition of the structure into nonoverlapping subdomains. FETI and BDD were initially developed for homogeneous structures, and later extended to heterogeneous structures using preconditioners [3] and initialization [4]. However when dealing with structures containing large heterogeneities and high contrast of phase properties, these methods often exhibit poor performance. More specifically, when the subdomain interface intersects the heterogeneities, the interface problem lacks an efficient preconditioner. Several techniques have been proposed to alleviate these issues such as FETI-Geneo (FETI-generalized eigenvalues in the overlaps) [5], multipreconditioned FETI method (MPFETI) [6] or MultiPreconditioned algorithm (AMPFETI)

${ }^{*}$ Corresponding author: J. Yvonnet, Université Paris-Est, Laboratoire Modélisation et Simulation Multi Echelle, MSME UMR 8208 CNRS, 5 bd Descartes, 77454 Marne la Vallée, France. E-mail: julien.yvonnet@univ-paris-est.fr 
[7. 8]. Another technique, the Large Time Increment (LATIN) method [9, 10], is a non-incremental iterative computational strategy where local (nonlinear) problems solutions in subdomains are updated through a global linear problem through interfaces conditions and an appropriate search direction to ensure the global convergence.

Other parallel strategies have been proposed, such as the algebraic multigrid method (AMG) [11, 12, 13, 14], developed to solve efficiently large linear system of equations. Such approach is a purely algebraic matrix-based approach used to deal with large sparse linear systems and can be considered as a solver to various finite element discretization problems without any geometrical or physical background. The main principle of AMG lies in the coarsening of a given linear system of equations using a coarse grid in order to reduce the problem size.

Multiscale methods constitute another class of approaches to solve the fully detailed heterogeneous structures. For example, in the method proposed by by Zohdi et al. (see e.g. [15], [16]), the concepts of homogenization are used in an iterative way. Each iteration consists in solving the homogeneous macro structure, using the effective elasticity tensor, which is computed from substructure problems. The macro strain solution is then applied to the substructure interfaces as Dirichlet boundary conditions and an interface corrector is used to minimize the interface error. Other multiscale methods or subdomain methods with appropriate reduction have been proposed, such as e.g. in [17, 18, 19, 20]. Another approach which has similarities with the method proposed in this work is the Multiscale Finite Element method [21, 22], where shape functions obtained from fine scale calculations in each coarse mesh elements substitute the polynomial shape functions. However, the treatments to reduce discontinuities across interfaces induce discontinuities in the displacement field in the reconstructed solution at the fine scale.

Another approach is the direct use of homogenization methods to include the effects of strain gradient in the case where scales are not separated. This situation occurs when the wavelength associated with the strain and stress fields at the micro scale is of the same order of magnitude as the wavelength of the prescribed loads or the characteristic dimensions of the structure. In such situations, first order computational homogenization (see e.g. [23]) fails to capture boundary effects or localized fields near defects. For these purposes, strain gradient computational homogenization approaches have been proposed, including Cosserat-type generalized continuum media [24, 25], second-order computational homogenization [26, 27, 28, 29], asymptotic expansion-based approaches [30], extended computational homogenization with more refined strain gradient fields [31] or nonlocal homogenization methods [32].

In this paper, we introduce a new multiscale method called Coarse Mesh Condensation Multiscale Method (CMCM), which shares analogies with the $\mathrm{FE}^{2}$ method but with aim to solve directly a heterogeneous structure. The $\mathrm{FE}^{2}$ method was originally proposed in [33, 34, 24]. In this method, the main idea is to solve a heterogeneous nonlinear structure problem by means of a macroscopic mesh, whose strain state defines boundary conditions on representative volume elements (RVE) associated to a lower scale, which after solving the local problem provides the macro stress through averaging. One appealing feature of $\mathrm{FE}^{2}$ is that the local (fine scale) problems can be solved in parallel, as being independent of each others. $\mathrm{FE}^{2}$ has been widely applied and extended to many problems including secondorder homogenization, transient conduction problems, model reduction, damage localization or topology optimization [27, 35, 36, 37, 38, 39].

In this work, we use analogies with this idea to solve heterogeneous structures without scale separation trough an efficient approximation of the fine scale solution by means of parallel computations on subdomains. Even though possibly applied to nonlinear problems, we restrict in this work to linear problems and show that in this case the technique leads to an efficient one-iteration scheme where local solution (in subdomains decomposing the structure) can be solved in parallel through off-line calculations. Here, the solution of fine scale subdomain problems are related to the degrees of freedom of a coarse mesh which has much fewer degrees of freedom than the full problem (by several order of magnitudes). The summary of the paper is as follows. In section 2, we first introduce notations used in this paper. In Section 3 we describe CMCM and provide practical application details in 2D and 3D. Numerical examples involving both academic validation tests as well as large industrial examples are presented and discussed in section 4 .

\section{Preliminary: notations}

Vectors and second order tensors, as well as matrices, are denoted by bold letters $\mathbf{A}$. Third order tensors are denoted by calligraphic uppercase letters $\mathcal{G}$, fourth-order, fifth-order and sixth-order tensors are denoted by double case letters $\mathbb{A}$. Double contraction of indices for second order tensors $\mathbf{A}$ and $\mathbf{B}$ is denoted by $\mathbf{A}: \mathbf{B}=A_{i j} B_{i j}$, dot product for two vectors $\mathbf{a}$ and $\mathbf{b}$ by $\mathbf{a} \cdot \mathbf{b}=a_{i} b_{i}$, and simple contraction of indices for a second order tensor $\mathbf{A}$ and a vector $\mathbf{b}$ is denoted by $(\mathbf{A b})_{i}=A_{i j} b_{j}$. The gradient operator is denoted by $\nabla($.$) and the divergence operator by \nabla \cdot($.$) .$ 


\begin{tabular}{|c|c|c|}
\hline $\mathbf{x}$ & : & Position vector at the fine scale \\
\hline $\mathbf{u}(\mathbf{x})$ & : & Displacement vector at the fine scale \\
\hline$\varepsilon$ & : & Linearized strain tensor \\
\hline$\sigma$ & : & Cauchy stress tensor \\
\hline $\mathcal{G}$ & : & Tensor of second gradient of displacements \\
\hline$\nabla \varepsilon$ & : & Strain gradient tensor \\
\hline $\mathbb{C}(\mathbf{x})$ & : & Elastic tensor \\
\hline $\mathbb{C}^{0}(\mathbf{x})$ & : & Auxiliary elastic tensor \\
\hline $\bar{\varepsilon}$ & : & Macroscopic linearized strain tensor \\
\hline$\overline{\mathcal{G}}$ & : & Macroscopic second gradient of displacements tensor \\
\hline$\nabla \bar{\varepsilon}$ & : & Macroscopic strain gradient tensor \\
\hline$\overline{\mathbf{g}}^{\alpha}$ & : & $\begin{array}{l}\text { Vector of parameters defining Dirichlet boundary conditions on the } \\
\text { boundary of } \Omega^{\alpha}\end{array}$ \\
\hline$\Omega^{\alpha}$ & : & Subdomain $\alpha$ \\
\hline $\mathbf{A}^{\alpha}(\mathbf{x})$ & : & Localization tensor in $\Omega^{\alpha}$ \\
\hline$[\varepsilon(\mathbf{x})]$ & : & Vector for associated with $\varepsilon$ \\
\hline$[\sigma(\mathbf{x})]$ & : & Vector form associated with $\sigma$ \\
\hline $\mathbf{C}(\mathbf{x})$ & : & Matrix form associated with $\mathbb{C}(\mathbf{x})$ \\
\hline$\Omega^{m}$ & : & Domain associated with an element $m$ in the fine mesh \\
\hline$\Omega^{e}$ & : & Domain associated with an element $e$ in the fine mesh \\
\hline $\mathbf{u}^{m}$ & : & Vector of nodal displacements in one element $m$ of the fine mesh \\
\hline$\overline{\mathbf{u}}^{e}$ & : & Vector of nodal displacements in one element $e$ of the coarse mesh \\
\hline
\end{tabular}

Table 1: Notations

Let $\mathbf{u}$ be defined as the displacement vector and $\mathbf{x}$ as a material coordinate, we define the linearized strain as:

$$
\varepsilon_{i j}=\frac{1}{2}\left(\frac{\partial u_{i}}{\partial x_{j}}+\frac{\partial u_{j}}{\partial x_{i}}\right),
$$

the second gradient displacement tensor by

$$
\mathcal{G}_{i j k}=\frac{\partial^{2} u_{i}}{\partial x_{j} \partial x_{k}}
$$

and the strain gradient tensor as:

$$
\nabla \varepsilon_{i j k}=\frac{1}{2}\left(\frac{\partial^{2} u_{i}}{\partial x_{j} \partial x_{k}}+\frac{\partial^{2} u_{j}}{\partial x_{i} \partial x_{k}}\right) .
$$

We denote the analogous macro quantities $\overline{\boldsymbol{\varepsilon}}, \overline{\mathcal{G}}$ and $\nabla \overline{\boldsymbol{\varepsilon}}$ as the macro strain, macro second gradient of displacements and macro strain gradient tensors, respectively. 


\section{Coarse Mesh Condensation Multiscale Method (CMCM)}

\subsection{General description}

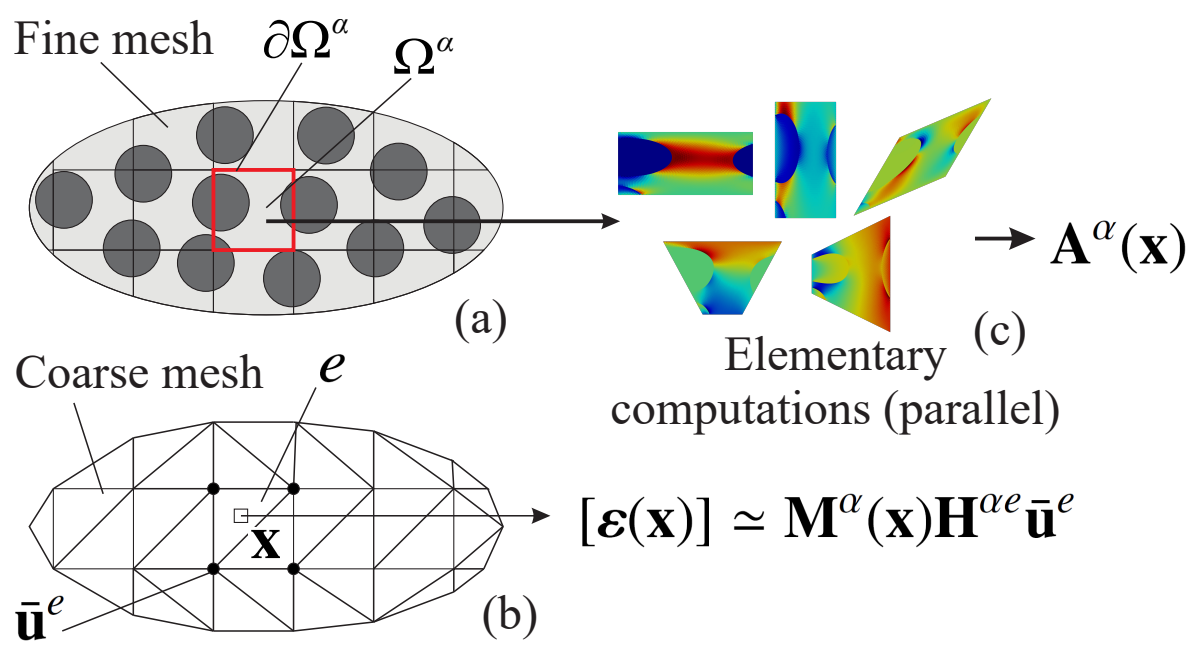

Figure 1: Schematic description of the CMCM method; (a) decomposition of the structure into subdomains; (b) discretization of the structure with a coarse mesh; (c) elementary (off-line) problems to be solved over the subdomains.

The key idea of CMCM approximation is to construct an approximation of the strain and stress solution fields at the finest scale in subdomains whose solutions can be computed in parallel through off-line calculations. First, the heterogeneous structure is decomposed into subdomains $\Omega^{\alpha}, \alpha=1,2, \ldots, N_{\text {sub }}$ where $N_{\text {sub }}$ denotes the number of subdomains, and such that $\Omega=\bigcup_{\alpha} \Omega^{\alpha}$ (see Fig. 1 (a)). Then, each subdomain is discretized with a mesh of finite elements associated with the fine scale. In addition, a coarse mesh is defined to discretize the structure, independently of the heterogeneities (see Fig. 1 (b)). Dirichlet boundary conditions are then defined over each subdomain, and associated with a small number of parameters, gathered in a vector $\overline{\mathbf{g}}^{\alpha}$ (see section 3.2). We define these parameters such that they minimize the error in the least square senses between the strain field in the subdomain and the strain field in the elements of the coarse mesh covering the subdomain. In the linear case, a linear relationship can be established between displacements of the coarse mesh and the strain field in the subdomains. Note that this approximation depends on the location of the point $\mathbf{x}$ in the element of the coarse mesh and is then richer than the simple linear combination of subdomain elementary solutions (see details in section 3.2.1). Then, the solution in the subdomains can be condensed at the nodes of the coarse mesh and the problem can be solved on this coarse mesh only to define the fine scale solution by post-treatment. The main steps are summarized below and described in more details in the following.

(i) Solve localization problems in each subdomain $\Omega^{\alpha}$ (parallel computations).

(ii) Determine the relationship between the dofs of the coarse mesh and the reduced vector $\overline{\mathbf{g}}^{\alpha}$ in each subdomain $\Omega^{\alpha}$ (parallel computations).

(iii) Solve the global problem on the coarse mesh.

(iv) Re-localization of fine scale fields from global problem solution.

\subsection{Step (i): Subdomain problem}

\subsubsection{Localization problem on subdomains}

We first define the problem to be solved on each individual subdomain $\Omega^{\alpha}$ whose boundary is denoted by $\partial \Omega^{\alpha}$ (see Fig. 11 (a). A general expression of Dirichlet boundary conditions is introduced as:

$$
u_{i}(\mathbf{x})=\sum_{k} \bar{g}_{k}^{\alpha} d_{i k}^{\alpha}(\mathbf{x}) \text { on } \partial \Omega^{\alpha},
$$


where $\overline{\mathbf{g}}^{\alpha}$ is the reduced parameters vector. The size of $\overline{\mathbf{g}}^{\alpha}$ is assumed to be small as compared to the total number of dofs on the boundary $\partial \Omega^{\alpha}$ in order to perform a reduced condensation of the internal dofs.

In this work, we adopt the particular form for relation (4) as:

$$
u_{i}(\mathbf{x})=\bar{\varepsilon}_{i j} x_{j}+\frac{1}{2} \overline{\mathcal{G}}_{i j k} x_{j} x_{k} \text { on } \partial \Omega^{\alpha},
$$

where $\bar{\varepsilon}_{i j}$ is a macroscopic strain tensor and $\overline{\mathcal{G}}_{i j k}$ is a macroscopic third-order second gradient displacement tensor, which can also be re-expressed as a function of an effective (homogeneous) first gradient of the strain tensor $\nabla \bar{\varepsilon}_{i j k}$ through (see e.g. [40, 41]):

$$
\overline{\mathcal{G}}_{i j k}=\nabla \bar{\varepsilon}_{i j k}+\nabla \bar{\varepsilon}_{i k j}-\nabla \bar{\varepsilon}_{j k i} .
$$

In addition, we restrict the approximation (5) to the $x_{j} x_{k},(j \neq k)$ terms to reduce the number of parameters, i.e. terms products of $x_{i}^{2}$ are set to zero. The corresponding obtained solutions will be referred to as Second-order CMCM in the example (section 4). For comparison, another approximation of (5) keeping only the linear terms (i.e. setting all $\bar{G}$ term products to zero) will be considered and referred to as $C M C M$ in the examples.

Special attention must be paid to the case of a homogeneous subdomain, i.e. containing only one material phase. In that situation, we must ensure that the boundary conditions (5) do not induce spurious fluctuations, i.e. that the solution in $\Omega^{\alpha}$ derives from $(5)$ in the form:

$$
\boldsymbol{\varepsilon}(\mathbf{x})=\bar{\varepsilon}+\nabla \bar{\varepsilon} \cdot \mathbf{x} \text { in } \Omega^{\alpha} .
$$

Considering that one subdomain contains a single material phase with elastic properties $\mathbb{C}^{0}$, applying the Hooke's law and taking the divergence of (7) one obtains:

which is verified if

$$
\nabla \cdot\left(\mathbb{C}^{0}: \boldsymbol{\varepsilon}(\mathbf{x})\right)=\nabla \cdot\left(\mathbb{C}^{0}:[\overline{\boldsymbol{\varepsilon}}+\nabla \overline{\boldsymbol{\varepsilon}} \cdot \mathbf{x}]\right)=\mathbf{f},
$$

$$
f_{i}=C_{i j k l}^{0} \nabla \bar{\varepsilon}_{k l j}
$$

Then, body forces must be prescribed in addition to the boundary conditions to ensure null fluctuations in the case of a homogeneous subdomain. One possible choice for $\mathbb{C}^{0}$ is (see [42]) $C_{i j k l}^{0}=\overline{\mathbb{C}}$, where $\overline{\mathbb{C}}$ is the homogenized elastic modulus defined by

$$
\overline{\mathbb{C}}=\frac{1}{V} \int_{\Omega^{\alpha}} \mathbb{C}(\mathbf{x}): \mathbb{A}(\mathbf{x}) d \Omega
$$

and where $\mathbb{A}(\mathbf{x})$ is the classical localization tensor. Then, the localization problem is defined as follows:

Given $\overline{\mathbf{g}}^{\alpha}=\{\overline{\boldsymbol{\varepsilon}}, \nabla \overline{\boldsymbol{\varepsilon}}\}$, find $\boldsymbol{\varepsilon}(\mathbf{x})$ in $\Omega^{\alpha}$ such that:

$$
\begin{gathered}
\nabla \cdot(\mathbb{C}(\mathbf{x}): \boldsymbol{\varepsilon}(\mathbf{x}))=\mathbf{f}, \\
f_{i}=\bar{C}_{i j k l} \nabla \bar{\varepsilon}_{k l j}, \\
\mathbf{u}(\mathbf{x})=\overline{\boldsymbol{\varepsilon}} \cdot \mathbf{x}+\frac{1}{2} \overline{\mathcal{G}}: \mathbf{x} \otimes \mathbf{x} \text { on } \partial \Omega^{\alpha} .
\end{gathered}
$$

We can see that with this definition, if the subdomain is homogeneous, then the solution is self-equilibrated for the above quadratic boundary conditions. Problem $[11-(13)$ is then classically solved by finite elements as follows.

The weak form of the problem to be solved on the subdomain $\Omega^{\alpha}$ is given by: search $\mathbf{u} \in H^{1}\left(\Omega^{\alpha}\right)$ satisfying the boundary conditions 13 and such that:

$$
\int_{\Omega^{\alpha}} \boldsymbol{\varepsilon}(\mathbf{u}): \mathbb{C}(\mathbf{x}): \boldsymbol{\varepsilon}(\delta \mathbf{u}) d \Omega=\int_{\Omega^{\alpha}} \mathbf{f}(\mathbf{x}) \cdot \delta \mathbf{u} d \Omega \quad \forall \delta \mathbf{u} \in H_{0}^{1}\left(\Omega^{\alpha}\right)
$$


where $H^{1}$ and $H_{0}^{1}$ are the usual Sobolev vector spaces. Introducing classical FEM discretization in (14), we obtain a linear system in the form:

$$
\mathbf{K}^{\alpha} \mathbf{u}=\mathbf{F}^{\alpha}
$$

with

$$
\mathbf{K}^{\alpha}=\int_{\Omega^{\alpha}} \mathbf{B}^{m T}(\mathbf{x}) \mathbf{C}(\mathbf{x}) \mathbf{B}^{m}(\mathbf{x}) d \Omega, \quad \mathbf{F}^{\alpha}=\int_{\Omega^{\alpha}} \mathbf{N}^{m T}(\mathbf{x}) \mathbf{f} d \Omega,
$$

where $\mathbf{N}^{*}$ and $\mathbf{B}^{m}$ are matrices of shape functions and of shape functions derivatives on the fine mesh in the subdomain, respectively (see Fig. 49, and $\mathbf{C}(\mathbf{x})$ is the matrix form associated with $\mathbb{C}(\mathbf{x})$. It is worth noting that for one subdomain $\Omega^{\alpha}$, the linear systems to be solved for each component of $\overline{\mathbf{g}}^{\alpha}$ involve the same matrix $\mathbf{K}^{\alpha}$, which thus has to be assembled only once.

In case the when subdomain boundaries cross the interfaces, large errors can be generated by the boundary conditions (13). In that case, an extension of the method consist in prescribing the boundary conditions on the external boundary $\partial \tilde{\Omega}^{\alpha}$ of an extended subdomain $\tilde{\Omega}^{\alpha}$ (see Fig. 2). However, the localization tensor is only evaluated in the initial subdomain $\Omega^{\alpha}$ ). We define a parameter $\beta=h_{\text {ext }} / L_{0}$, where $L_{0}$ is the initial length of the subdomain $\Omega^{\alpha}$ (considered as square subdomain in this work for the sake of simplicity). Note that this process is similar to the oversampling technique in MSFEM [21].

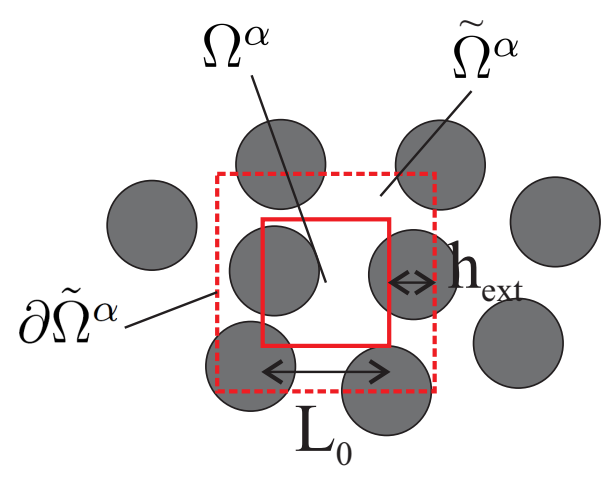

Figure 2: Illustration of an extended subdomain.

\subsubsection{Reduced parameters $\overline{\mathbf{g}}^{\alpha}$ in the $2 D$ case}

In $2 \mathrm{D}$, after neglecting terms in $x_{i}^{2}$, (13) leads to:

$$
\begin{aligned}
& u_{1}(\mathbf{x})=\bar{\varepsilon}_{11} x_{1}+\bar{\varepsilon}_{12} x_{2}+\frac{1}{2}\left(\overline{\mathcal{G}}_{112}+\overline{\mathcal{G}}_{121}\right) x_{1} x_{2}, \\
& u_{2}(\mathbf{x})=\bar{\varepsilon}_{12} x_{1}+\bar{\varepsilon}_{22} x_{2}+\frac{1}{2}\left(\overline{\mathcal{G}}_{212}+\overline{\mathcal{G}}_{221}\right) x_{1} x_{2} .
\end{aligned}
$$

After simplifications, we obtain:

$$
\begin{aligned}
& u_{1}(\mathbf{x})=\bar{\varepsilon}_{11} x_{1}+\bar{\varepsilon}_{12} x_{2}+\nabla \overline{\boldsymbol{\varepsilon}}_{112} x_{1} x_{2}, \\
& u_{2}(\mathbf{x})=\bar{\varepsilon}_{12} x_{1}+\bar{\varepsilon}_{22} x_{2}+\nabla \overline{\boldsymbol{\varepsilon}}_{221} x_{1} x_{2} .
\end{aligned}
$$

In the Second-order CMCM case, the boundary conditions of each $\Omega^{\alpha}$ subdomain are parameterized by 5 independent scalar values gathered in the $\overline{\mathbf{g}}^{\alpha}$ vector:

$$
\overline{\mathbf{g}}^{\alpha}=\left[\bar{\varepsilon}_{11}, \bar{\varepsilon}_{22}, \bar{\varepsilon}_{12}, \nabla \bar{\varepsilon}_{112}, \nabla \bar{\varepsilon}_{221}\right]^{T}
$$


As the problem to be solved over $\Omega^{\alpha}$ is linear, applying the superposition principle allows the strain field to be expressed in $\Omega^{\alpha}$ as:

$$
[\boldsymbol{\varepsilon}(\mathbf{x})]=\mathbf{A}^{\alpha}(\mathbf{x}) \overline{\mathbf{g}}^{\alpha} \quad \forall \mathbf{x} \in \Omega^{\alpha} .
$$

where $[\boldsymbol{\varepsilon}(\mathbf{x})]$ is the column-vector notation associated with the second-order strain tensor $\boldsymbol{\varepsilon}=\frac{1}{2}\left(\nabla \mathbf{u}+\nabla^{T} \mathbf{u}\right)$ and $\mathbf{A}^{\alpha}(\mathbf{x})$ is a localization matrix (see section 3.2.2).

The matrix $\mathbf{A}^{\alpha}(\mathbf{x})$ is therefore a $3 \times 5$ matrix whose columns $\left[\boldsymbol{\varepsilon}^{i}(\mathbf{x})\right]$ are the strain vector solution of $(11)-(13)$ for $\bar{g}_{i}^{\alpha}=1$ and $\bar{g}_{j}^{\alpha}=0, i \neq j$ :

$$
\mathbf{A}^{\alpha}(\mathbf{x})=\left[\left[\varepsilon^{1}(\mathbf{x})\right],\left[\varepsilon^{2}(\mathbf{x})\right],\left[\varepsilon^{3}(\mathbf{x})\right],\left[\varepsilon^{4}(\mathbf{x})\right],\left[\varepsilon^{5}(\mathbf{x})\right]\right] .
$$

In the basic CMCM case, only the first three terms of $\overline{\mathbf{g}}^{\alpha}$ and consequently the first three columns of $\mathbf{A}^{\alpha}$ are computed.

\subsubsection{Reduced parameters $\overline{\mathbf{g}}^{\alpha}$ in the $3 D$ case}

In $3 \mathrm{D}$, using (13) and (6), we obtain, after simplifications and neglecting terms in $x_{i}^{2}$ :

$$
\begin{aligned}
& u_{1}(\mathbf{x})=\bar{\varepsilon}_{11} x_{1}+\bar{\varepsilon}_{12} x_{2}+\bar{\varepsilon}_{13} x_{3}+\nabla \bar{\varepsilon}_{112} x_{1} x_{2}+\nabla \bar{\varepsilon}_{113} x_{1} x_{3}+\overline{\mathcal{G}}_{123} x_{2} x_{3}, \\
& u_{2}(\mathbf{x})=\bar{\varepsilon}_{12} x_{1}+\bar{\varepsilon}_{22} x_{2}+\bar{\varepsilon}_{23} x_{3}+\nabla \bar{\varepsilon}_{221} x_{1} x_{2}+\nabla \bar{\varepsilon}_{223} x_{2} x_{3}+\overline{\mathcal{G}}_{213} x_{1} x_{3}, \\
& u_{3}(\mathbf{x})=\bar{\varepsilon}_{13} x_{1}+\bar{\varepsilon}_{23} x_{2}+\bar{\varepsilon}_{33} x_{3}+\nabla \bar{\varepsilon}_{331} x_{1} x_{3}+\nabla \bar{\varepsilon}_{332} x_{2} x_{3}+\overline{\mathcal{G}}_{312} x_{1} x_{2} .
\end{aligned}
$$

In the Second-order CMCM case, $\overline{\mathbf{g}}^{\alpha}$ contains 15 independent parameters (and in the basic CMCM case, only the first 6 are needed):

$$
\overline{\mathbf{g}}^{\alpha}=\left[\bar{\varepsilon}_{11}, \bar{\varepsilon}_{22}, \bar{\varepsilon}_{33}, \bar{\varepsilon}_{12}, \bar{\varepsilon}_{13}, \bar{\varepsilon}_{23}, \nabla \bar{\varepsilon}_{112}, \nabla \bar{\varepsilon}_{113}, \nabla \bar{\varepsilon}_{221}, \nabla \bar{\varepsilon}_{223}, \nabla \bar{\varepsilon}_{331}, \nabla \bar{\varepsilon}_{332}, \overline{\mathcal{G}}_{123}, \overline{\mathcal{G}}_{213}, \overline{\mathcal{G}}_{312}\right]^{T} .
$$

Thus 15 (resp. 6) linear problems need to be solved on each subdomain. Moreover all these problems can be solved in parallel. The matrix $\mathbf{A}^{\alpha}(\mathbf{x})$ is in that case a $6 \times 15$ (resp. $\left.6 \times 6\right)$ matrix.

It is worth noting that in the case where the strain gradient parameters are applied, the subdomain needs to be centered in order to obtain the correct deformation mode.

\subsection{Step (ii): Relation between coarse mesh-displacements and subdomain boundary conditions}

To relate the solutions in the subdomains to the coarse mesh, a relationship between the vector of boundary conditions $\overline{\mathbf{g}}^{\alpha}$ of subdomain $\Omega^{\alpha}$ and the dofs in one element $\Omega^{e}$ of the coarse mesh, denoted by $\overline{\mathbf{u}}^{e}$, must be established. For this purpose, we minimize the distance between the strain approximation provided by (22) and the strain in each element of the coarse mesh individually (see Fig. 1), which is given by:

$$
[\hat{\boldsymbol{\varepsilon}}(\mathbf{x})]=\overline{\mathbf{B}}^{e}(\mathbf{x}) \overline{\mathbf{u}}^{e},
$$

where $\overline{\mathbf{B}}^{e}$ is a matrix of shape functions derivatives of one element $\Omega^{e}$ of the coarse mesh. Then the problem to be solved is given by

$$
\overline{\mathbf{g}}^{\alpha}=\operatorname{Arg} \min J
$$

with

$$
J=\int_{\Omega^{e}}\left(\mathbf{A}^{\alpha}(\mathbf{x}) \overline{\mathbf{g}}^{\alpha}-\overline{\mathbf{B}}^{e} \overline{\mathbf{u}}^{e}\right)^{2} d \Omega
$$


where $\Omega^{e}$ is the domain associated with the element $e$ in the coarse mesh, and $(\mathbf{a})^{2}=\mathbf{a} \cdot \mathbf{a}$, a being a vector. Minimizing $J$ with respect to $\overline{\mathbf{g}}^{\alpha}$ gives (writing $\bar{g}_{i}^{\alpha} \equiv g_{i}, A_{i j}^{\alpha} \equiv A_{i j}, \bar{B}_{i j}^{e} \equiv B_{i j}$ and $\bar{u}_{i n c}^{E} \equiv u_{i}$ for alleviating the notations):

$$
\frac{\partial J}{\partial g_{m}}=0, \quad m=1,2, \ldots, N_{g}
$$

or;

$$
\int_{\Omega^{e}} 2 A_{i j} \frac{\partial g_{j}}{\partial g_{m}}\left(A_{i j} g_{j}-B_{i j} u_{j}\right) d \Omega=0 .
$$

Then:

$$
\int_{\Omega^{e}} A_{i m}(\mathbf{x}) A_{i j}(\mathbf{x}) d \Omega g_{j}=\int_{\Omega^{e}} A_{i m}(\mathbf{x}) B_{i j}(\mathbf{x}) u_{j} d \Omega \quad m=1,2, \ldots, N_{g} .
$$

We obtain a linear system of equations in the form:

$$
\mathbf{G}^{\alpha} \overline{\mathbf{g}}^{\alpha}=\mathbf{H}^{\alpha e} \overline{\mathbf{u}}^{e}
$$

and then:

$$
\overline{\mathbf{g}}^{\alpha}=\left(\mathbf{G}^{\alpha}\right)^{-1} \mathbf{H}^{\alpha e} \overline{\mathbf{u}}^{e},
$$

with:

$$
\mathbf{G}^{\alpha}=\int_{\Omega^{\alpha}}\left(\mathbf{A}^{\alpha}(\mathbf{x})\right)^{T} \mathbf{A}^{\alpha}(\mathbf{x}) d \Omega
$$

and

$$
\mathbf{H}^{\alpha e}=\int_{\Omega^{e}}\left(\mathbf{A}^{\alpha}(\mathbf{x})\right)^{T} \overline{\mathbf{B}}^{e}(\mathbf{x}) d \Omega .
$$

Finally, we obtain the approximation of the fine scale strain in each element $\Omega^{e}$ of the coarse mesh covering a subdomain $\Omega^{\alpha}$, using (22) and (33), as:

$$
[\boldsymbol{\varepsilon}(\mathbf{x})]=\mathbf{A}^{\alpha}(\mathbf{x})\left(\mathbf{G}^{\alpha}\right)^{-1} \mathbf{H}^{\alpha e} \overline{\mathbf{u}}^{e}=\mathbf{M}^{\alpha}(\mathbf{x}) \mathbf{H}^{\alpha e} \overline{\mathbf{u}}^{e},
$$

or

$$
[\boldsymbol{\varepsilon}(\mathbf{x})]=\mathbf{M}^{\alpha}(\mathbf{x}) \mathbf{H}^{\alpha e} \overline{\mathbf{u}}^{e}
$$

with

$$
\mathbf{M}^{\alpha}(\mathbf{x})=\mathbf{A}^{\alpha}(\mathbf{x})\left(\mathbf{G}^{\alpha}\right)^{-1} .
$$

\subsection{Step (iii): Global problem}

We now consider the problem to be solved on the coarse mesh over the entire structure (see Fig. 11(b)). Assuming zero body forces, the governing equations of the problem in the structure are given by:

$$
\begin{cases}\nabla \cdot \sigma(\mathbf{x})=0 & \forall \mathbf{x} \in \Omega, \\ \sigma(\mathbf{x})=\mathbb{C}(\mathbf{x}): \boldsymbol{\varepsilon}(\mathbf{x}) & \forall \mathbf{x} \in \Omega, \\ \mathbf{u}(\mathbf{x})=\overline{\mathbf{u}}^{*} & \forall \mathbf{x} \in \partial \Omega_{u}, \\ \sigma \cdot \mathbf{n}=\overline{\mathbf{F}}^{*} & \forall \mathbf{x} \in \partial \Omega_{F},\end{cases}
$$

where $\overline{\mathbf{u}}^{*}$ and $\overline{\mathbf{F}}^{*}$ are prescribed displacements and tractions on the corresponding Dirichlet and Neumann boundaries $\partial \Omega_{u}$ and $\partial \Omega_{F}$. Using vector forms of strain tensors, the weak form of the system of equations 440 can be expressed as follows:

$$
\int_{\Omega}[\varepsilon(\mathbf{u})]^{T} \mathbf{C}(\mathbf{x})[\varepsilon(\delta \mathbf{u})] d \Omega=\int_{\partial \Omega_{F}} \overline{\mathbf{F}}^{*} \cdot \delta \mathbf{u} d \Gamma .
$$


Considering the same approximation for test functions $\delta \overline{\mathbf{u}}^{e}$ as in 38 :

$$
[\boldsymbol{\varepsilon}(\delta \mathbf{u})]=\mathbf{M}^{\alpha}(\mathbf{x}) \mathbf{H}^{\alpha e} \delta \overline{\mathbf{u}}^{e},
$$

and substituting (38) and (45) in (44) we obtain:

$$
\delta \overline{\mathbf{u}}^{e T} \sum_{\alpha} \int_{\Omega^{\alpha}}\left(\mathbf{H}^{\alpha e}\right)^{T}\left(\mathbf{M}^{\alpha}(\mathbf{x})\right)^{T} \mathbf{C}(\mathbf{x}) \mathbf{M}^{\alpha}(\mathbf{x}) \mathbf{H}^{\alpha e} d \Omega \overline{\mathbf{u}}^{e}=\delta \overline{\mathbf{u}}^{e T} \int_{\partial \Omega_{F}} \mathbf{N}^{T}(\mathbf{x}) \mathbf{F}^{*} d \Gamma .
$$

Owing to the arbitrariness of $\delta \overline{\mathbf{u}}^{T T}$, we then obtain a linear system of equations in the form:

$$
\overline{\mathbf{K}} \overline{\mathbf{u}}=\overline{\mathbf{F}}
$$

where:

$$
\overline{\mathbf{K}}=\sum_{\alpha} \int_{\Omega^{\alpha}}\left(\mathbf{H}^{\alpha e}\right)^{T}\left(\mathbf{M}^{\alpha}(\mathbf{x})\right)^{T} \mathbf{C}(\mathbf{x}) \mathbf{M}^{\alpha}(\mathbf{x}) \mathbf{H}^{\alpha e} d \Omega .
$$

In practice, $\overline{\mathbf{K}}$ is assembled from elementary matrices computed in each micro element $m$ covered by an element $e$ defined in its associated domain $\Omega^{e}$ of the coarse mesh (see Fig. 3). Given the subdomain $\Omega^{\alpha}$ containing the fine mesh element $e^{m}$, the matrix $\mathbf{H}^{\alpha e}$ is constant in $\Omega^{\alpha}$ and then the elementary matrix $\overline{\mathbf{K}}$ can be expressed as:

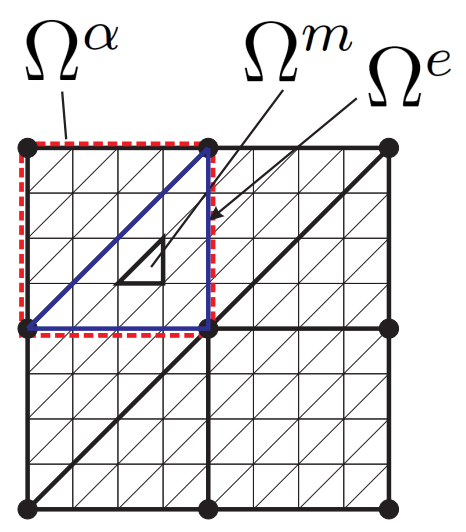

Figure 3: Domain $\Omega^{\alpha}$, coarse mesh element $e$ associated with a domain $\Omega^{e}$ and fine mesh element $m$ associated with a domain $\Omega^{m}$.

$$
\overline{\mathbf{K}}^{m}=\left(\mathbf{H}^{\alpha e}\right)^{T} \int_{\Omega^{m}}\left(\mathbf{M}^{\alpha}(\mathbf{x})\right)^{T} \mathbf{C}(\mathbf{x}) \mathbf{M}^{\alpha}(\mathbf{x}) d \Omega \mathbf{H}^{\alpha e}
$$

where $\Omega^{m}$ is the domain associated with one fine mesh element $m$ and

$$
\overline{\mathbf{F}}=\int_{\partial \Omega_{F}} \overline{\mathbf{N}}^{T}(\mathbf{x}) \overline{\mathbf{F}}^{*} d \Gamma,
$$

where $\overline{\mathbf{N}}$ is the shape function matrix of elements in the coarse mesh. The Dirichlet and Neumann boundary conditions are classically prescribed on the coarse mesh. Once $\overline{\mathbf{u}}$ is known, the strain field on the fine mesh in each subdomain can be reconstructed by (34) and the stress using:

$$
[\boldsymbol{\sigma}(\mathbf{x})]=\mathbf{C}(\mathbf{x}) \mathbf{M}^{\alpha}(\mathbf{x}) \mathbf{H}^{\alpha e} \overline{\mathbf{u}}^{e} .
$$

In this work, we did not consider the case when one micro element $m$ cut the boundaries of the domain $\Omega^{e}$ occupied by a coarse mesh element. This point is reported to future studies. The algorithm for solving the coarse mesh problem is summarized in Algorithm 2. 

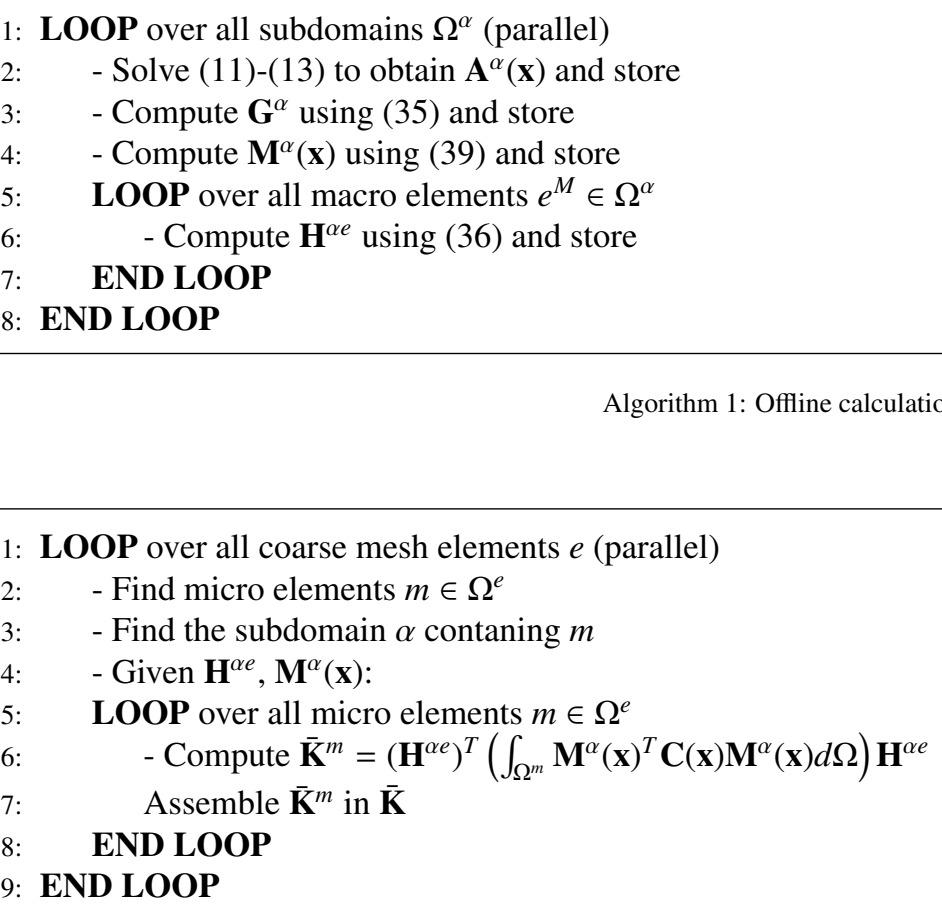

\subsection{Analogies between $F E^{2}$ and $C M C M$}
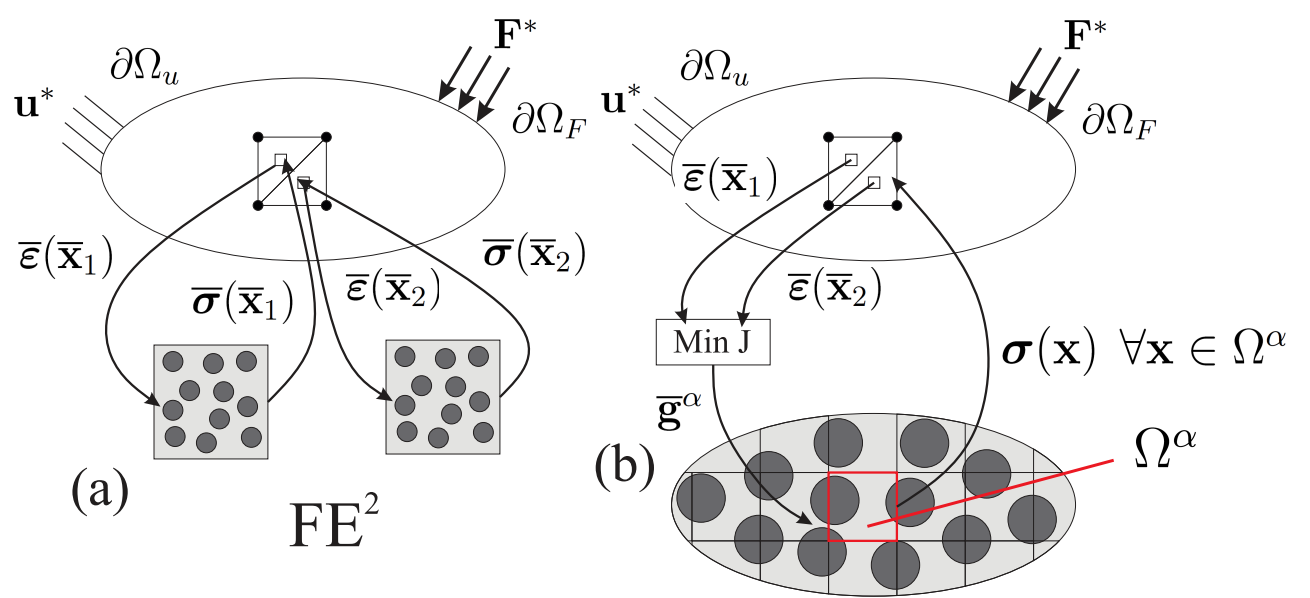

$\mathrm{CMCM}$

Figure 4: (a) Schematic description of $\mathrm{FE}^{2}$ method [33] and of CMCM.

In this subsection, we show analogies between the present method and the $\mathrm{FE}^{2}$ method, originally proposed in [24] to solve nonlinear multiscale problems with separated scales. In $\mathrm{FE}^{2}$ (see Fig. 49 (a)), a relationship between macro strain $\overline{\boldsymbol{\varepsilon}}$ and the macro stress $\overline{\boldsymbol{\sigma}}$ is obtained numerically at each Gauss point of the coarse mesh by: (i) prescribing Dirichlet boundary conditions depending on $\bar{\varepsilon}$ on the boundary of an RVE associated with the element; (ii) solving the local problem on the RVE; (iii) averaging the stress in the RVE to obtain $\bar{\sigma}$. In CMCM (see Fig. 49 (b)), a group of elements is covering a subdomain. Solving a minimization problem (see section 3.3), Dirichlet boundary 
conditions are prescribed on the boundary of the subdomain. The solution is then solved on the subdomain to obtain the stress field. The main differences are summarized as follows: (a) in $\mathrm{FE}^{2}$, the local calculations are computed on an RVE associated with the microstructure. In CMCM, the local calculations are performed in subdomains whose union is the fully detailed heterogeneous structure; (b) $\mathrm{In} \mathrm{FE}^{2}$, the parameters defining the boundary conditions on the local problem (RVE) are associated with a single integration point in the mesh at the maccroscale. In CMCM, the parameters associated with boundary conditions to be applied on the local problem (subdomain) depend on the strain field of all elements of the coarse mesh covering the subdomain; (c) in $\mathrm{FE}^{2}$, the local problem is used to define the macro strain -macro stress relationship. In CMCM, the local problem is used to relate the strain in the coarse mesh to the full stress field in all elements in the subdomain (fine scale) covered by the coarse mesh elements and the notion of macro stress is no more necessary. Both methods share the following features: (a) all local computations can be performed in parallel; (b) the local solution (fine scale) can be reconstructed given the macro (coarse) mesh solution; (c) the macro (coarse) mesh problem involves much fewer degrees of freedom as compared to solving the full problem.

However it is necessary to make it clear that CMCM is not a special case of $\mathrm{FE}^{2}$. Even though the method can be potentially applied to nonlinear problems, we only treat linear problems in this paper. The treatment of nonlinear problems would involve the same idea but applied to the linearized problem in a Newton solving procedure. In such, the appealing parallel solving feature of the method would be maintained. However, the developments related to nonlinear problems are out of the scope of the present work and are reported to future studies.

As another remark, note that in CMCM the full microstructure has to be known everywhere in the structure, in contrast with $\mathrm{FE}^{2}$. However, we recall that this method applies to structures where scale cannot be separated, and where the size of heterogeneities becomes not negligible as compared to the size of the structure, as for example in woven composites. In industrial application, it is nowadays common to obtain microtomography images of the full structure up to the level of the yarns.

\section{Numerical examples}

In this section, we analyze and demonstrate the potential of the method through both academic and simplified industrial examples of realistic scale. In the present work we only consider periodic heterogeneous structures, even though the technique is not restricted to this class of problems. The application to non-repeatable structures will be discussed in a separate study. In the context of periodic subdomains, the local problem (11)-(13) has to be solved only once (see section 3.2). In the following, three solutions are considered for comparison:

- A reference solution obtained by a direct finite element calculation, where the structure is fully meshed, covering all the details of the heterogeneities. The reference solution of the largest 3D problem is obtained with the iterative AMPFETI method [7, 8].

- A solution using the present method without using strain gradient (denoted by CMCM).

- A solution using the present method with strain gradient (denoted by CMCM).

An energy global error is defined as:

$$
\operatorname{Err}^{E}=\frac{\int_{\Omega}\left(\boldsymbol{\varepsilon}^{r e f}(\mathbf{x})-\boldsymbol{\varepsilon}^{C M C M}(\mathbf{x})\right): \mathbb{C}(\mathbf{x}):\left(\boldsymbol{\varepsilon}^{r e f}(\mathbf{x})-\boldsymbol{\varepsilon}^{C M C M}(\mathbf{x})\right) d \Omega}{\int_{\Omega} \boldsymbol{\varepsilon}^{r e f}(\mathbf{x}): \mathbb{C}(\mathbf{x}): \boldsymbol{\varepsilon}^{r e f}(\mathbf{x}) d \Omega}
$$

where $\varepsilon^{r e f}$ denotes the strain field obtained by the reference solution, and $\varepsilon^{C M C M}$ denotes the corresponding strain field obtained from CMCM.

Alternatively, a global $L_{2}$-error is defined as:

$$
E r r^{L 2}=\frac{\int_{\Omega}\left(\mathbf{u}^{r e f}(\mathbf{x})-\mathbf{u}^{C M C M}(\mathbf{x})\right) \cdot\left(\mathbf{u}^{r e f}(\mathbf{x})-\mathbf{u}^{C M C M}(\mathbf{x})\right) d \Omega}{\int_{\Omega}\left(\mathbf{u}^{r e f}(\mathbf{x})\right) \cdot\left(\mathbf{u}^{r e f}(\mathbf{x})\right) d \Omega}
$$

where $\mathbf{u}^{\text {ref }}$ denotes the displacement field obtained by the reference solution, and $\mathbf{u}^{C M C M}$ denotes the corresponding displacement field obtained from CMCM. 


\subsection{D periodic composite structure with cylindrical fibers}

We consider the academic structure depicted in Fig. 5(a), containing 4 periodic cylindrical fibers. Here, the structure is subdivided into 4 subdomains (see Fig. 9 (b)). The size of the square structure is $L=180 \mathrm{~mm}$, and the diameter of the fibers is $D=54 \mathrm{~mm}$. The matrix is assumed to be isotropic elastic with coefficients $E_{\text {mat }}=1$ Mpa and $v_{\text {mat }}=0.25$. The inclusions are also isotropic, with Poisson's ration $v_{i n c}=0.25$. We analyze several values of the inclusion Young modulus: $E_{i n c}=5 \mathrm{MPa}$, which is roughly the ratio of phase properties found in typical ceramics/ceramics composites, $E_{i n c}=10^{3} \mathrm{MPa}$ (large ratio) and an extreme case $E_{i n c}=10^{6} \mathrm{MPa}$ to analyze the capabilities of the method in that case, and especially in the case when the subdomains cross the interfaces, as such case is known to be a difficult one for iterative FETI domain decomposition methods (see [5]). The structure is subjected to the following Dirichlet boundary conditions:

$$
\mathbf{u}(\mathbf{x})=\left\{\begin{array}{l}
u_{x}=\frac{x^{2} y}{\alpha} \\
u_{y}=-\frac{x^{3}}{3 \alpha}
\end{array} \quad \text { on } \partial \Omega,\right.
$$

where $\alpha=10^{6} \mathrm{~mm}^{2}$ and $\partial \Omega$ is the whole external boundary of the structure. We first solve the problem with the present method without the strain gradient enhancement (CMCM). The reference finite element mesh describing the entire structure including all heterogeneities is composed of 73130 linear triangular elements, corresponding to 73852 degrees of freedom.

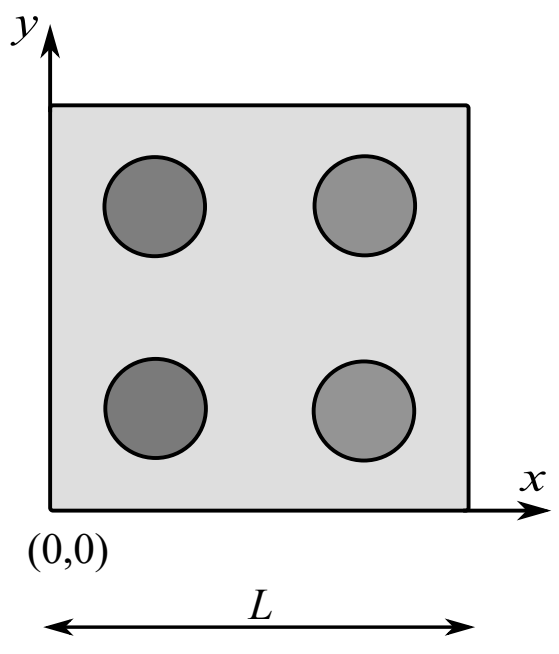

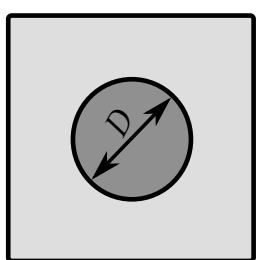

(b) A subdomain

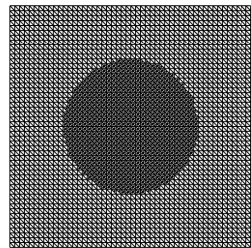

(c) Subdomain mesh

(a) Structure geometry

Figure 5: (a) Composite structure: geometry; (b) subdomain used for off-line calculations; (c) subdomain finite element mesh.

In order to investigate the convergence of the proposed method, 2 cases will be considered in this example:

- For a fixed number of subdomains, we vary the number of coarse mesh elements.

- For a fixed number of coarse mesh elements, we vary the number of subdomains.

\subsubsection{Convergence with respect to the number of coarse mesh elements}

In this first case, we fix the number of subdomains to 4 and increase the number of coarse mesh elements to analyze the convergence of the error. The case $E_{i n c}=10^{6} \mathrm{MPa}$ is first chosen (most defavorable case). In this work, we use 4-node bilinear elements and 8-node trilinear elements in 2D and 3D respectively for the coarse mesh. Five coarse meshes are used, whose numbers of elements and dofs are provided in Table 2.

Fig. 6 shows a comparison of the strain field $\varepsilon_{11}(\mathbf{x})$ solution obtained with CMCM with the 5 meshes and the reference solution. We can note that the local solution, reconstructed from (34), is significantly improved when the coarse mesh is refined. 
In Figs. 7 and 8 , the global energy and $L_{2}$ errors are plotted as a function of the number of coarse mesh dofs, and for several values of $\beta$, the parameter which defines the size of the oversampling (see section 3.2.1). We can see from Figs. 7 and 8 that the convergence is observed with both respect to the number of coarse mesh dofs and to $\beta$.

\begin{tabular}{cccccc}
\hline & Coarse mesh 1 & Coarse mesh 2 & Coarse mesh 3 & Coarse mesh 4 & Coarse mesh 5 \\
\hline Number of elements & 4 & 16 & 64 & 256 & 1024 \\
\hline Number of dofs & 18 & 50 & 162 & 578 & 2178 \\
\hline
\end{tabular}

Table 2: Number of elements and dofs for each coarse mesh.

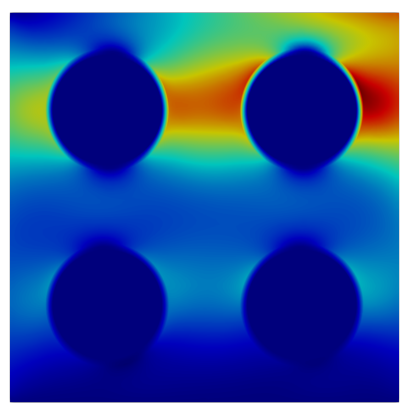

(a) Reference solution

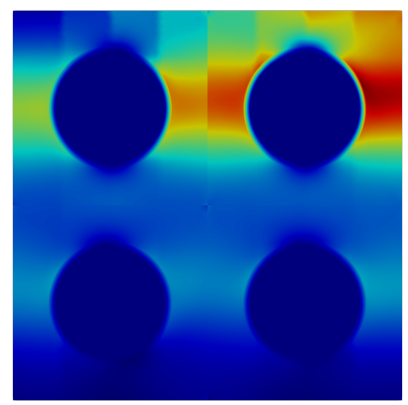

(d) Coarse mesh 3

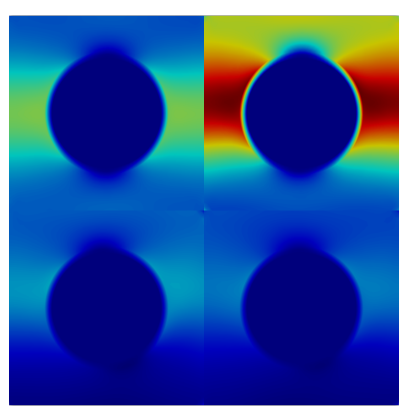

(b) Coarse mesh 1

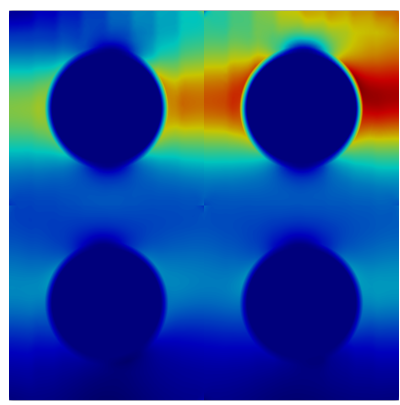

(e) Coarse mesh 4

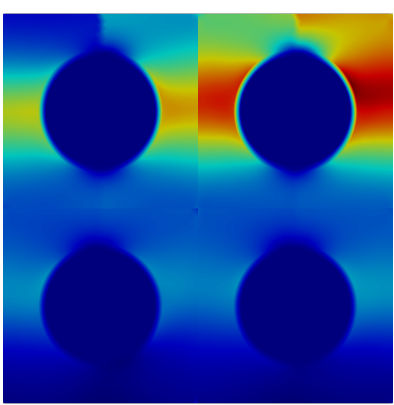

(c) Coarse mesh 2

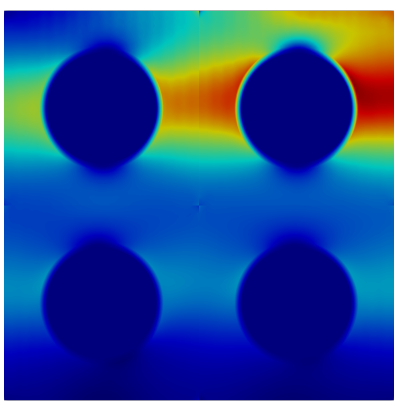

(f) Coarse mesh 5

$$
\varepsilon_{11}(\mathbf{x})
$$

$\begin{array}{lllllllll}-1.5 e-03 & 0.01 & 0.02 & 0.03 & 0.04 & 0.05 & 0.06 & 0.07 & 8.1 e-02\end{array}$

Figure 6: $\varepsilon_{11}(\mathbf{x})$ of reference and different coarse meshes. 


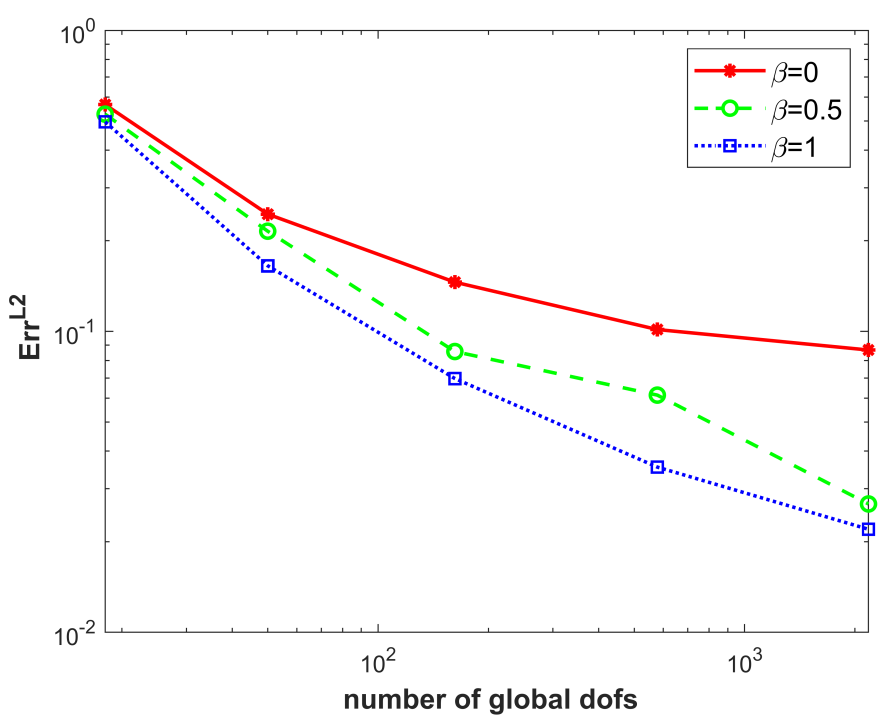

Figure 8: Evolution of $L_{2}$ error with respect to the number of coarse mesh dofs.

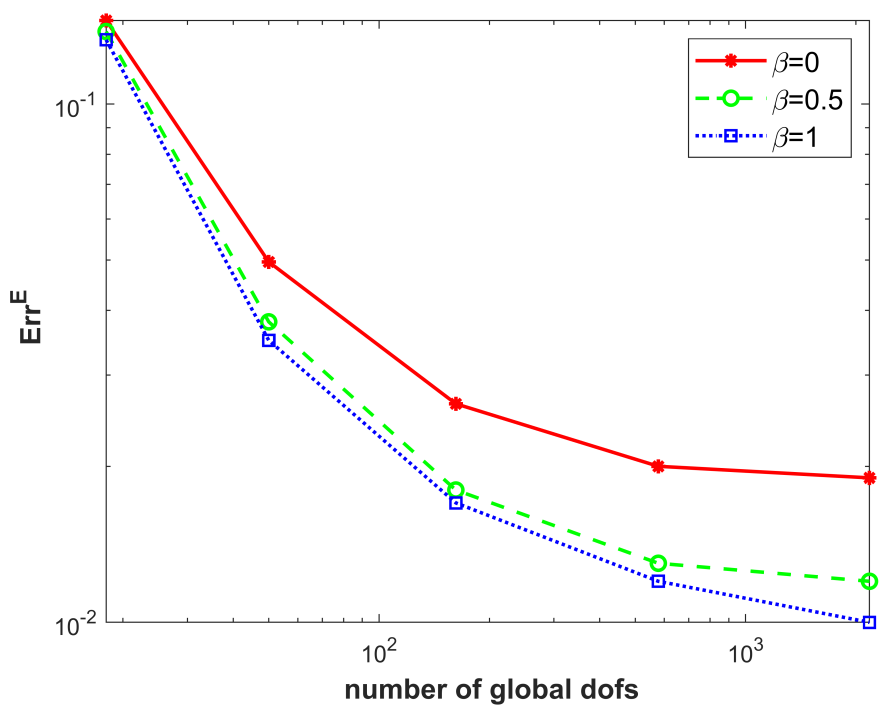

Figure 7: Evolution of energy error with respect to the number of coarse mesh dofs.

\subsubsection{Case 2: fixing number of coarse mesh dofs, changing the number of subdomains}

In this case, the number of coarse mesh elements is fixed and equal to 502, corresponding to 1305 dofs. The number of subdomains is then increased as described in Fig. 9 In the case of 16 subdomains, we can note that the interfaces cross the inclusions.

In this test, we investigate the influence of $\beta$ on the relocalized solution for the case with 16 subdomains (Fig. 9 (c)). Several values of $\beta(\beta=0.16,0.33,0.47,0.82$ and 1) are used. Figs. 11 and 12 show the stress fields with respect to $\beta$ in the cases $E_{i n c}=10^{6} \mathrm{MPa}$ and $E_{\text {inc }}=5 \mathrm{MPa}$. The evolution of global energy error and $L_{2}$ error versus $\beta$ is given in Fig. 10. We can see that the obtained global errors are very high when the interfaces cross the inclusions (i.e. $\beta=0.16,0.33$ and 0.47 ). However, when the extended subdomains cover the whole inclusions (i.e. $\beta=0.82$ and 
1), the global errors are significantly reduced. Error also decrease with respect to the phase properties contrast. More interestingly, for contrasts of the order of 5 (as e.g. in ceramics/ceramics composites), the error is low even for $\beta=0$.

We now use the converged solution in the case of 16 subdomains (i.e. $\beta=1$ ) and compare to the cases of 1 and 4 subdomains. Fig. 13 (a), (b), (c) and (d) show the relocalized stress field of the refence solution and of the CMCM solution, for 1, 4 and 16 subdomains, respectively in the case $E_{i n c}=10^{6} \mathrm{MPa}$ and in Figs. 14 in the case $E_{i n c}=5$ MPa. Fig. 15] shows the evolution of global strain energy error in function of number of subdomains. We can see that when the number of subdomains increases, the corresponding global error increases. This is due to the fact that some errors are localized at the interfaces between subdomains, thus more subdomains we have, more errors are likely to be committed. However, it is worth noting that in the third case where interface crosses inclusions, the global error is 0.032 which is still reasonable considering a high contrast between inclusion and matrix properties $\left(10^{6}\right)$.

In conclusion, from the numerical results, we can see that refining the coarse mesh indeed reduces the global error while increasing the number of subdomain increases the global error, due to the fact that the chances that the subdomains cross the interfaces are higher. When inclusions cut the interfaces we have seen that the use of the larger subdomains $(\beta>0)$ (see section 3.2.1) might drastically reduce the errors. If this case can be avoided, e.g. by means of appropriate choices of subdomains not crossing the interfaces, the errors can also be reduced. These errors also reduce with the contrast of phase properties between the inclusions and the matrix. it is also worth noting that defining an error indicator in this framework and an associate mesh adaptation would be useful and might deserve investigations in future works.

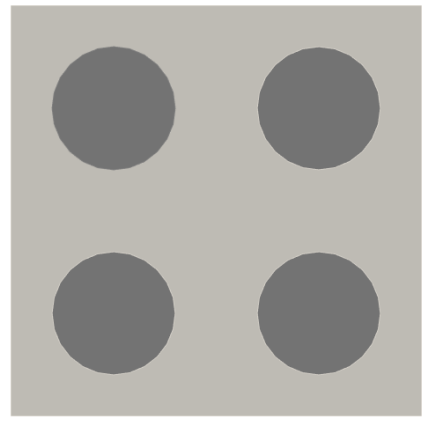

(a) 1 subdomain

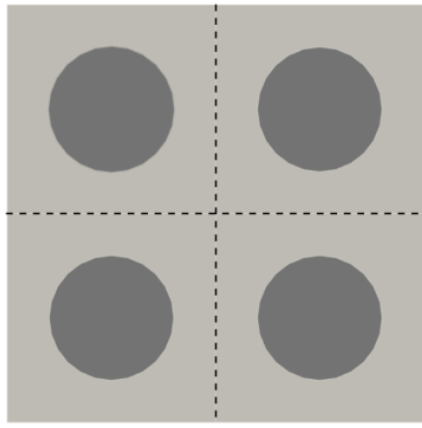

(b) 4 subdomains

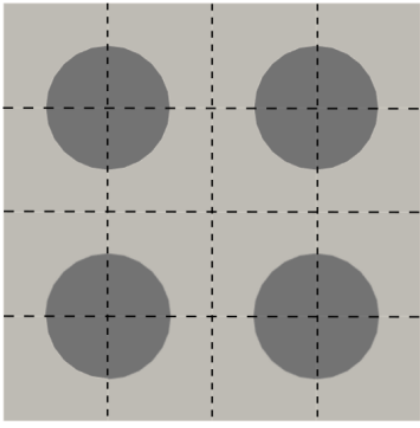

(c) 16 subdomains

Figure 9: Structure divided into (a) 1 subdomain, (b) 4 subdomains and (c) 16 subdomains.

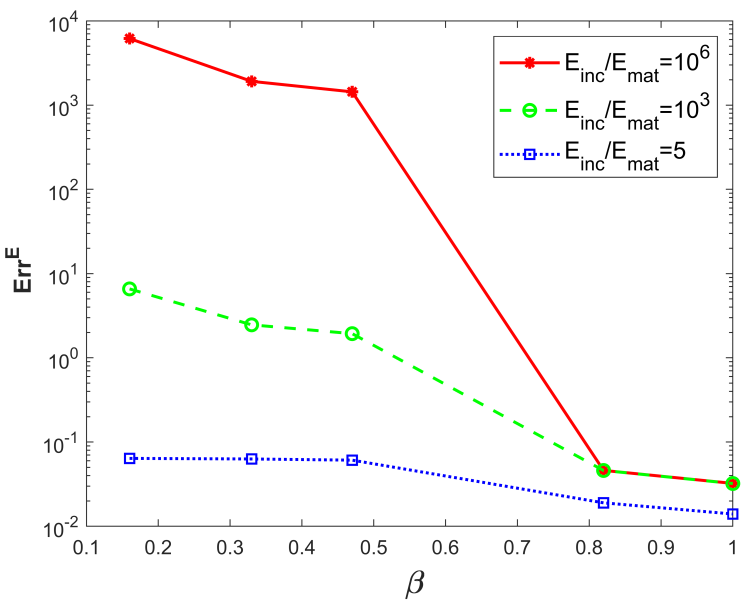

(a) Energy error

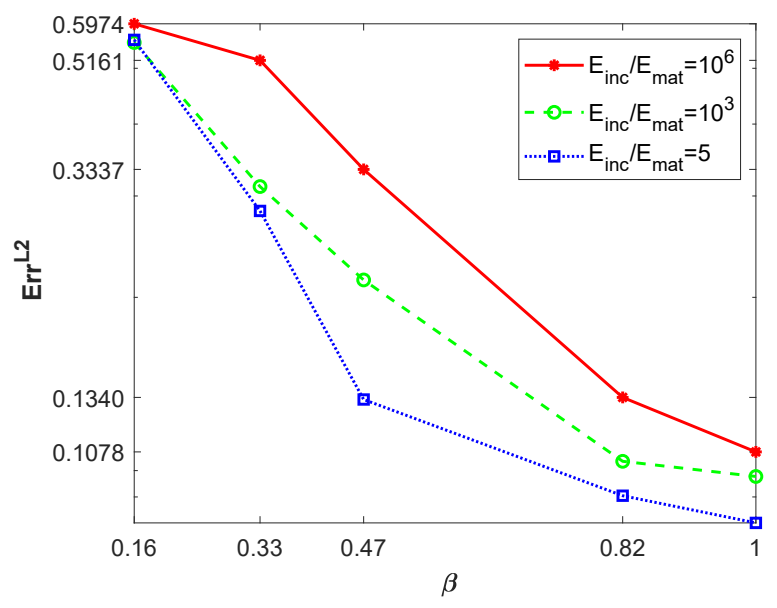

(b) $L_{2}$ error

Figure 10: Evolution of energy error (left) and $L_{2}$ error (right) in function of different values of $\beta$ in the case of 16 subdomains with contrast $\mathrm{E}_{\mathrm{inc}} / \mathrm{E}_{\mathrm{mat}}=10^{6}, 10^{3}$ and 5 . 


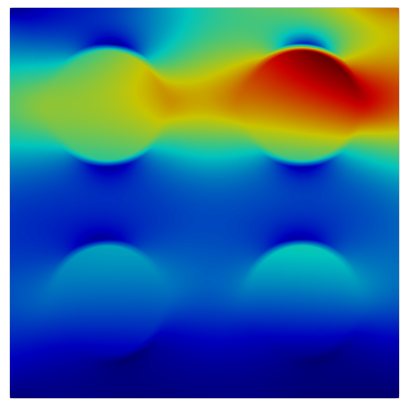

(a) Reference solution

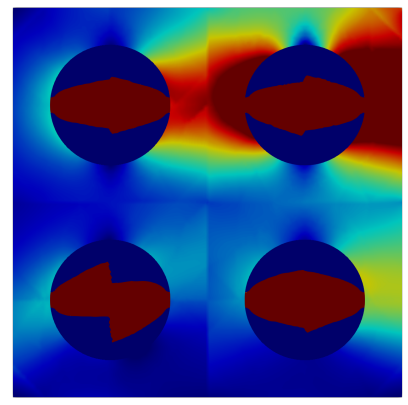

(d) $\beta=0.47$

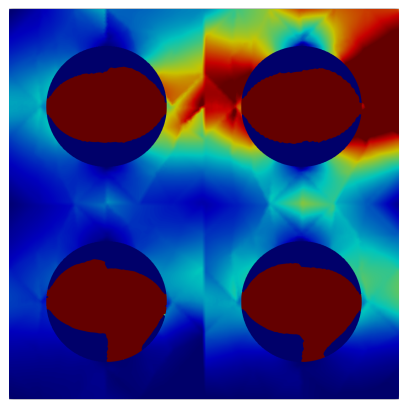

(b) $\beta=0.16$

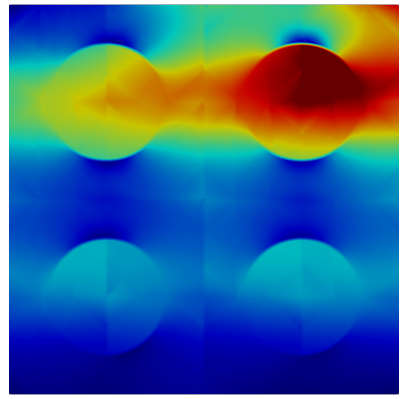

(e) $\beta=0.82$

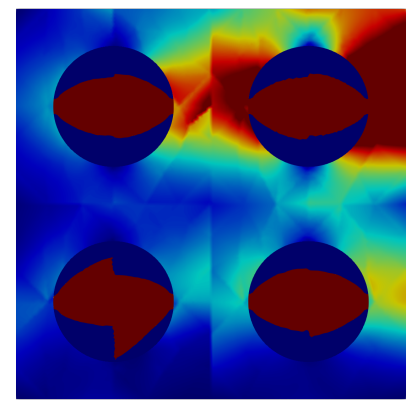

(c) $\beta=0.32$

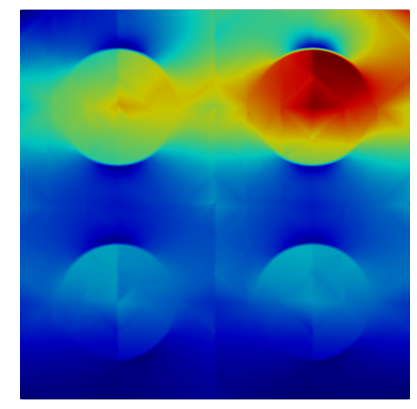

(f) $\beta=1$

$$
\sigma_{11}(\mathbf{x})(\mathrm{MPa})
$$

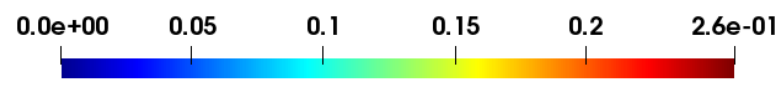

Figure 11: $\sigma_{11}(\mathbf{x})$ using different values of $\beta$ in the case of 16 subdomains with $\mathrm{E}_{\text {inc }} / \mathrm{E}_{\text {mat }}=10^{6}$. 


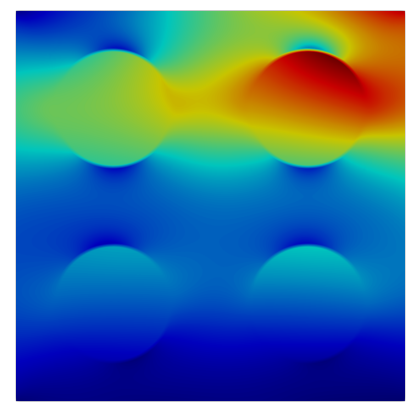

(a) Reference solution

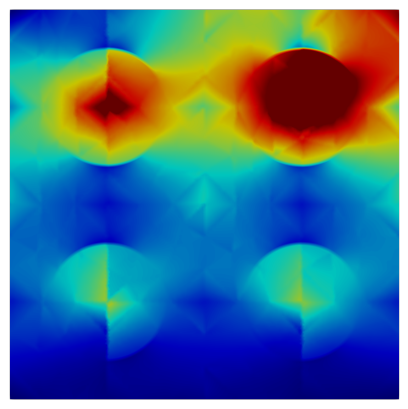

(d) $\beta=0.47$

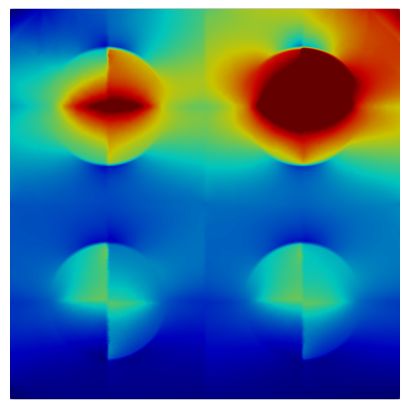

(b) $\beta=0.16$

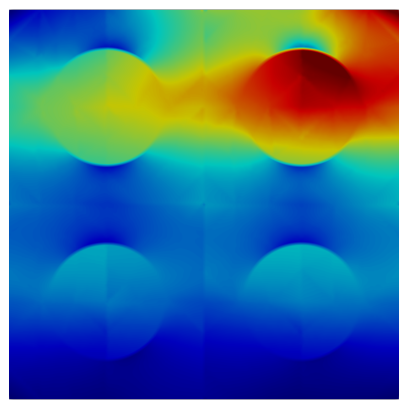

(e) $\beta=0.82$

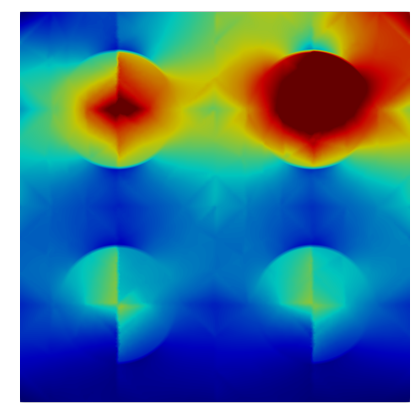

(c) $\beta=0.32$

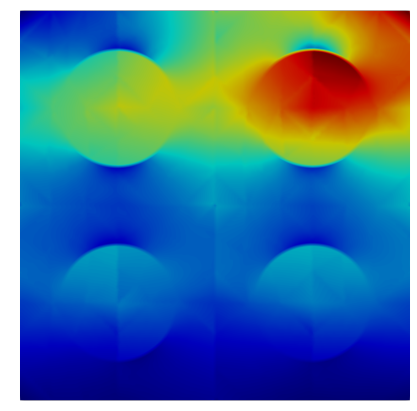

(f) $\beta=1$

$$
\sigma_{11}(\mathbf{x})(\mathrm{MPa})
$$

$\begin{array}{llllllllll}0.0 e+00 & 0.04 & 0.06 & 0.08 & 0.1 & 0.12 & 0.14 & 0.16 & 0.18 & 2.1 e-01\end{array}$

Figure 12: $\sigma_{11}(\mathbf{x})$ using different values of $\beta$ in the case of 16 subdomains with $\mathrm{E}_{\mathrm{inc}} / \mathrm{E}_{\mathrm{mat}}=5$. 


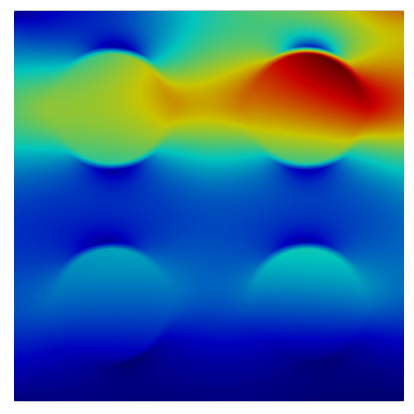

(a) Reference solution

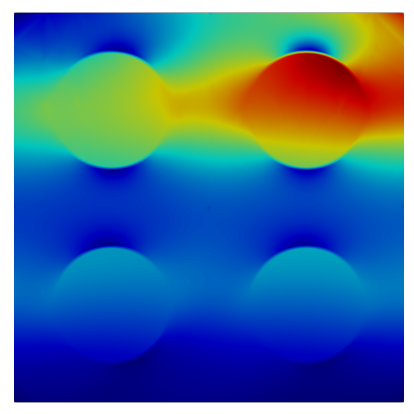

(c) 4 subdomains

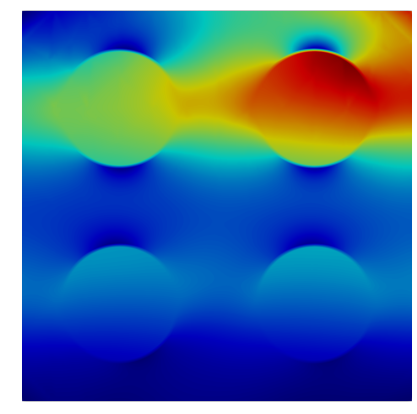

(b) 1 subdomain

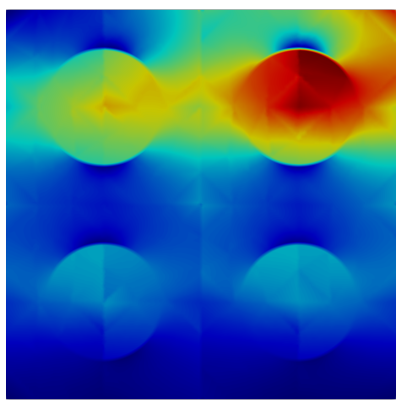

(d) 16 subdomains

$$
\sigma_{11}(\mathbf{x})(\mathrm{MPa})
$$

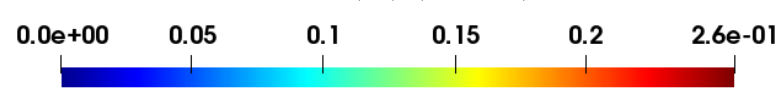

Figure 13: Relocalized stress $\sigma_{11}(\mathbf{x})(\mathrm{MPa}) \mathrm{E}_{\mathrm{inc}} / \mathrm{E}_{\mathrm{mat}}=10^{6}$ : (a) reference solution, (b) proposed methos with 1 subdomain, (c) proposed method with 4 subdomains and $(\mathrm{d})$ proposed method with 16 subdomains. 


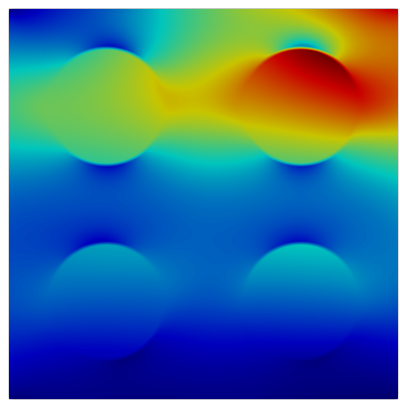

(a) Reference solution

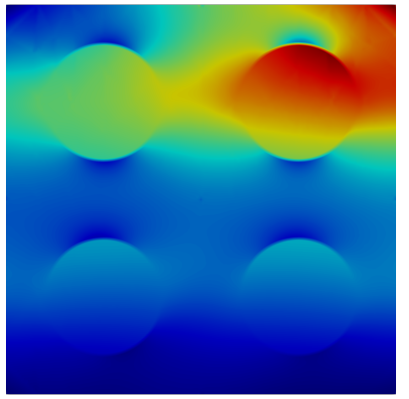

(c) 4 subdomains

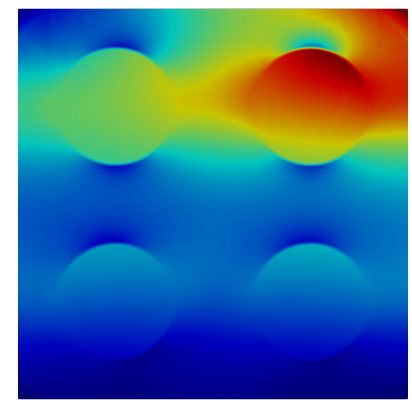

(b) 1 subdomain

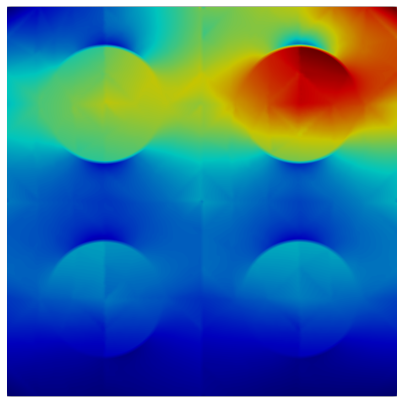

(d) 16 subdomains

$$
\sigma_{11}(\mathbf{x})(\mathrm{MPa})
$$

$\begin{array}{llllllllll}0.0 e+00 & 0.04 & 0.06 & 0.08 & 0.1 & 0.12 & 0.14 & 0.16 & 0.18 & 2.1 e-01\end{array}$

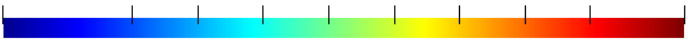

Figure 14: Relocalized stress $\sigma_{11}(\mathbf{x})(\mathrm{MPa})$ with $\mathrm{E}_{\text {inc }} / \mathrm{E}_{\text {mat }}=10^{3}$ : (a) reference solution, (b) proposed methos with 1 subdomain, (c) proposed method with 4 subdomains and (d) proposed method with 16 subdomains.

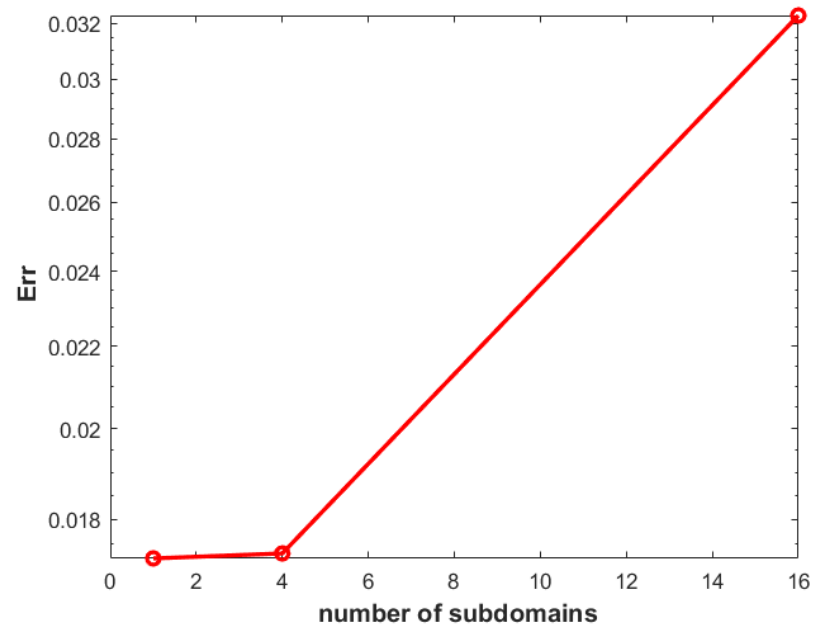

Figure 15: Evolution of energy error in function of number of subdomains. 


\subsubsection{Comparison with a simple method using fine integration and submodelling}

In this section, we compare the present method with a simple other approach using a coarse mesh and relocalization. In this method, the same fine mesh is defined over the whole domain to describe the microscale. The same coarse mesh as in Fig. 1 (a) is used. Here, the problem is first solved on the coarse mesh, but an exact integration is performed on the fine mesh. Then, a "submodelling" step is performed, by applying the obtained coarse mesh strain field as first order boundary conditions $\mathbf{u}(\mathbf{x})=\hat{\boldsymbol{\varepsilon}} \cdot \mathbf{x}$ (see Eq. 27 for the expression of $\hat{\boldsymbol{\varepsilon}}$ ) on a domain of fine mesh elements contained in the corresponding coarse mesh element, in order to obtain the displacement as well as strain and stress fields at the micro scale. It is worth noting that this simple approach does not require preliminary calculations and thus takes much less computational times. To perform fair comparisons, an extended subdomain method, as described in Fig. 2 is employed. Results are presented in Figs. 16, 17 and 18 The same example as in the previous section is chosen with a coarse mesh containing 256 elements (see Table 2). The contrast between inclusion and matrix properties is taken as $E_{\text {inc }} / E_{\text {mat }}=10^{6}$. Fig. 16 shows the evolution of the global error in the energy norm and $L_{2}$ error as a function of $\beta$, which defines the size of the extended subdomain. It can be shown that the error can be reduced from CMCM as comparison whith such simple approach. Figs. 17 and 18 show re-localized strain and stress fields obtained by both approaches for $\beta=1$, showing that the simple approach based on fine integration on the fine mesh is not able to capture correctly local fields while the present CMCM provides acceptable re-localized fine scale fields.

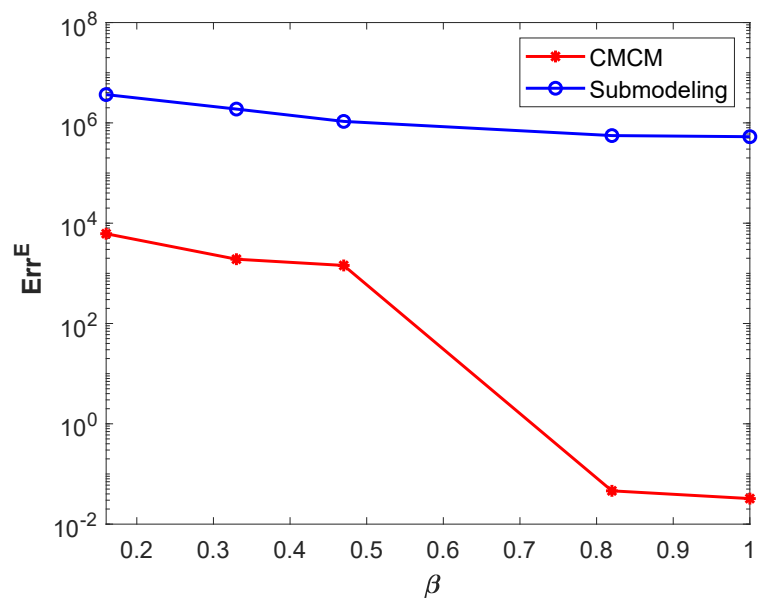

(a) Energy error

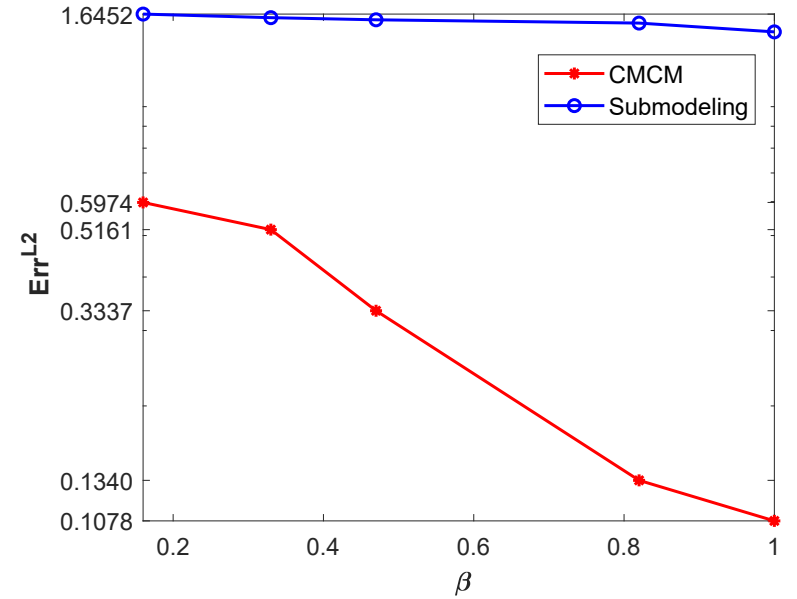

(b) $L_{2}$ error

Figure 16: Evolution of energy error (left) and $L_{2}$ error (right) in function of different values of $\beta$ with contrast $\mathrm{E}_{\mathrm{inc}} / \mathrm{E}_{\mathrm{mat}}=10^{6}$ using $\mathrm{CMCM}$ and submodeling. 


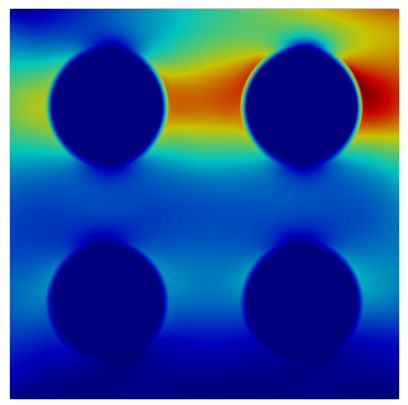

(a) Reference solution

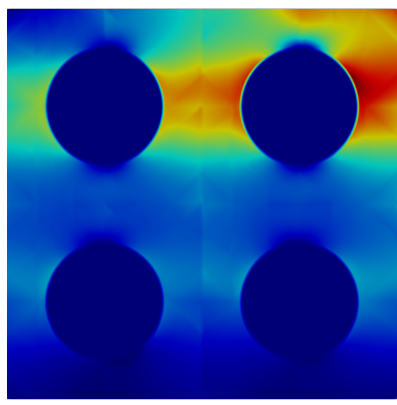

(b) $\mathrm{CMCM}$

$$
\varepsilon_{11}(\mathbf{x})
$$

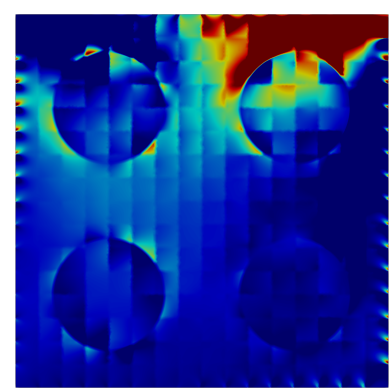

(c) Submodeling

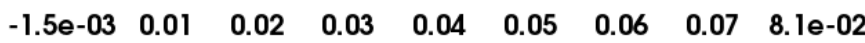

Figure 17: $\varepsilon_{11}(\mathbf{x})$ with $\mathrm{E}_{\mathrm{inc}} / \mathrm{E}_{\mathrm{mat}}=10^{6}$ of: (a) reference solution, (b) CMCM solution and (c) submodelling solution.

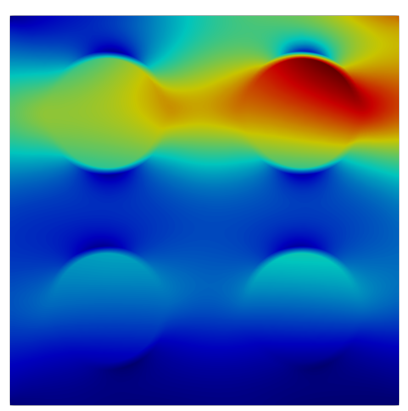

(a) Reference solution

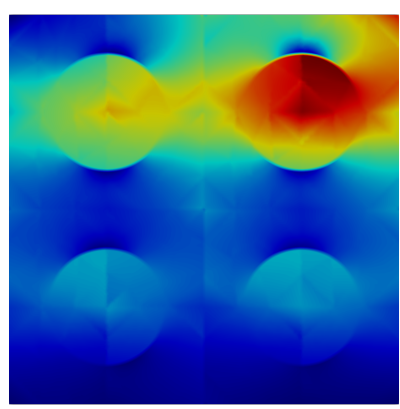

(b) $\mathrm{CMCM}$

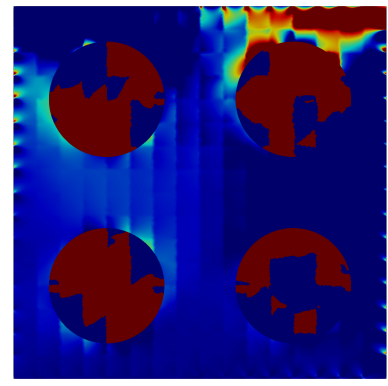

(c) Submodeling

$$
\sigma_{11}(\mathbf{x})(\mathrm{MPa})
$$

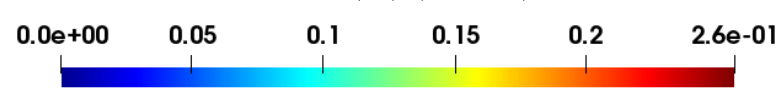

Figure 18: $\sigma_{11}(\mathbf{x})$ with $\mathrm{E}_{\text {inc }} / \mathrm{E}_{\text {mat }}=10^{6}$ of: (a) reference solution, (b) CMCM solution and (c) submodelling solution. 


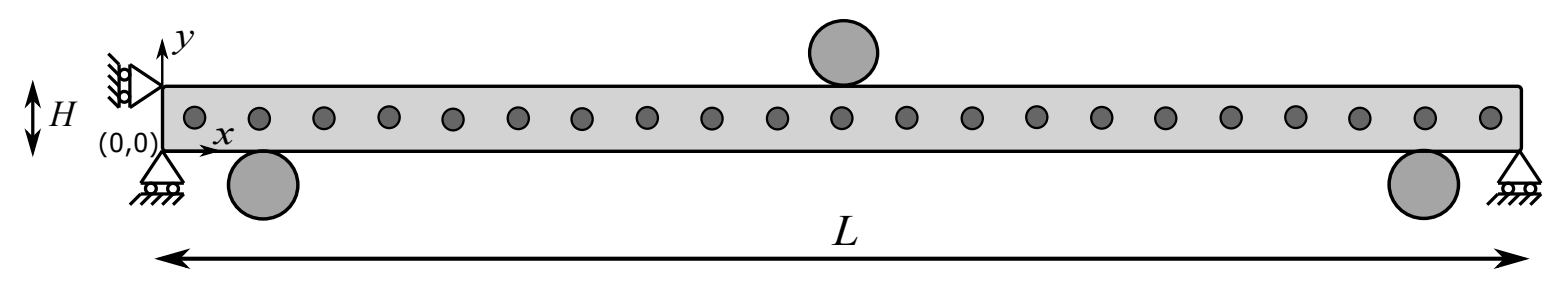

(a) Structure and boundary conditions

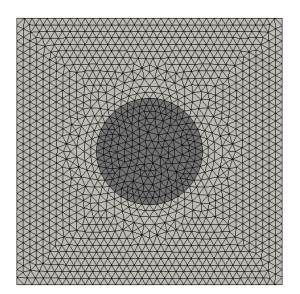

(b) Subdomain mesh

Figure 19: (a) 2D three-point bending composite beam: geometry and boundary conditions (b) subdomain finite element mesh.

In this example, we consider a composite beam under bending (see Fig. 19a). The objective of this academic example is to appreciate the gain obtained by introducing the strain gradient effects. The dimensions of the beam are $L=21 \mathrm{~mm}$ and $H=1 \mathrm{~mm}$. Each fiber has a diameter of $0.4 \mathrm{~mm}$ and is positioned at the center of a square subdomain of size $H$, as depicted in Fig. 19b. The mechanical properties of fibers and matrix are the same as in the previous example. The applied loading is a pressure field mimicking the reaction force of contact with cylinders. The corresponding fields are applied in the vicinity of three points with coordinates $\left(x_{i}^{c}, y_{i}^{c}\right)$ from left to right as follows: $(0.5,0),(10.5,1)$ and $(20.5,0)$ and are provided as:

$$
p_{i}(x)=p_{i}^{0}\left(1-\left(\frac{x-x_{i}^{c}}{x^{p}}\right)^{2}\right),
$$

where $p_{2}^{0}=20 \mathrm{MPa}, p_{1}^{0}=p_{3}^{0}=10 \mathrm{MPa}$ and $x^{p}=1 \mathrm{~mm}$. To remove rigid body motions, the nodal $y$-displacements at nodes $(0,0)$ and $(L, 0)$, and the nodal $x$-displacement at node $(0, H)$ are blocked. Three different coarse meshes are considered (Fig. 20). The number of elements and the corresponding number of dofs are listed in Table 3 ).

\begin{tabular}{ccccc}
\hline & subdomain & Coarse mesh 1 & Coarse mesh 2 & Coarse mesh 3 \\
\hline Element type & T3 & Q4 & Q4 & Q4 \\
\hline Number of elements & 5000 & 21 & 84 & 336 \\
\hline Number of dofs & 5202 & 88 & 258 & 850 \\
\hline
\end{tabular}

Table 3: Number of elements and of dofs for each mesh used in calculation (T3: linear triangular element; Q4: bilinear quadrilateral element). 


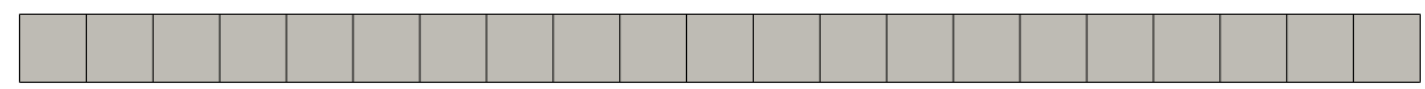

(a) Coarse mesh 1

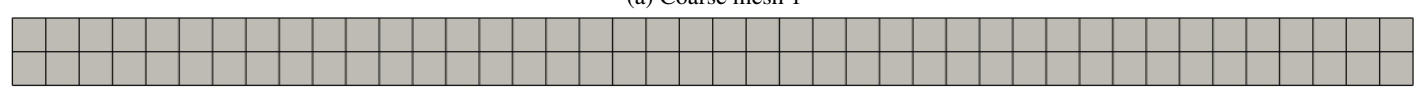

(b) Coarse mesh 2

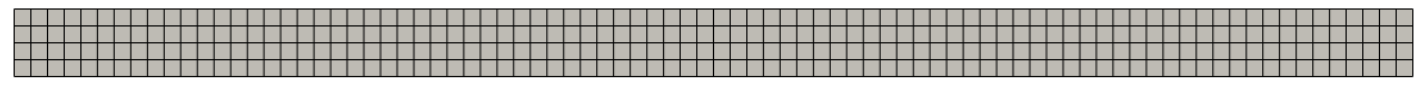

(c) Coarse mesh 3

Figure 20: Coarse meshes for global calculation of the beam: (a) coarse mesh 1, (b) coarse mesh 2, and (c) coarse mesh 3.

(a) Reference

(b) $\mathrm{CMCM}$

(c) Second-order CMCM

$\varepsilon_{11}(\mathbf{x})$

$\begin{array}{lllllllll}-0.21 & -0.15 & -0.10 & -0.050 & 0.00 & 0.050 & 0.10 & 0.15 & 0.21\end{array}$

Figure 21: Relocalized strain solution $\varepsilon_{11}(\mathbf{x})$

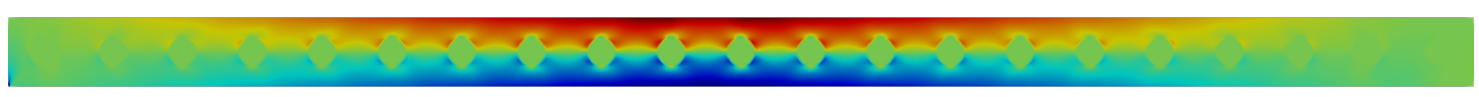

(a) Reference

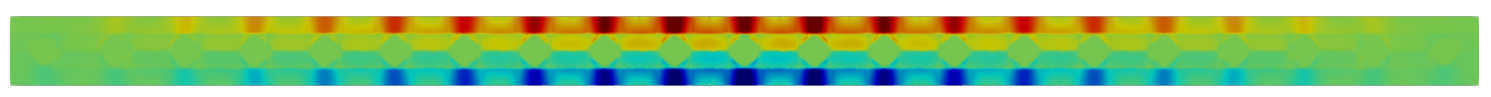

(b) $\mathrm{CMCM}$

(c) Second-order CMCM

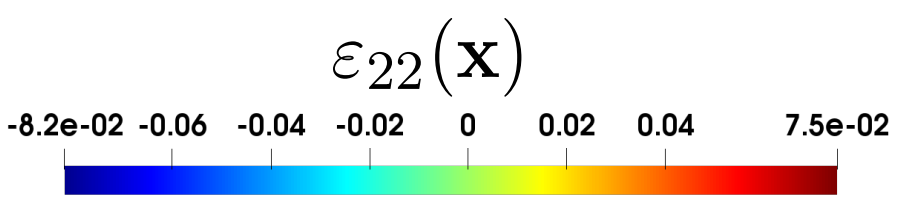

Figure 22: Relocalized strain solution $\varepsilon_{22}(\mathbf{x})$. 


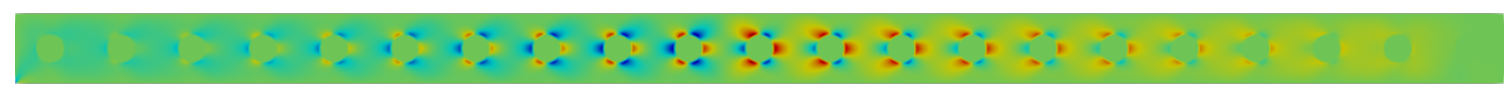

(a) Reference

(b) $\mathrm{CMCM}$

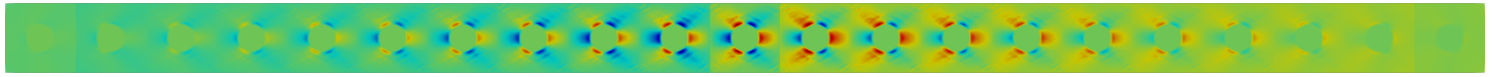

(c) Second-order CMCM

\section{$\varepsilon_{12}(\mathbf{x})$}

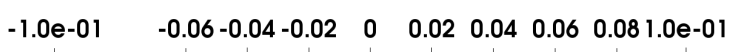

Figure 23: Relocalized strain solution $\varepsilon_{12}(\mathbf{x})$.

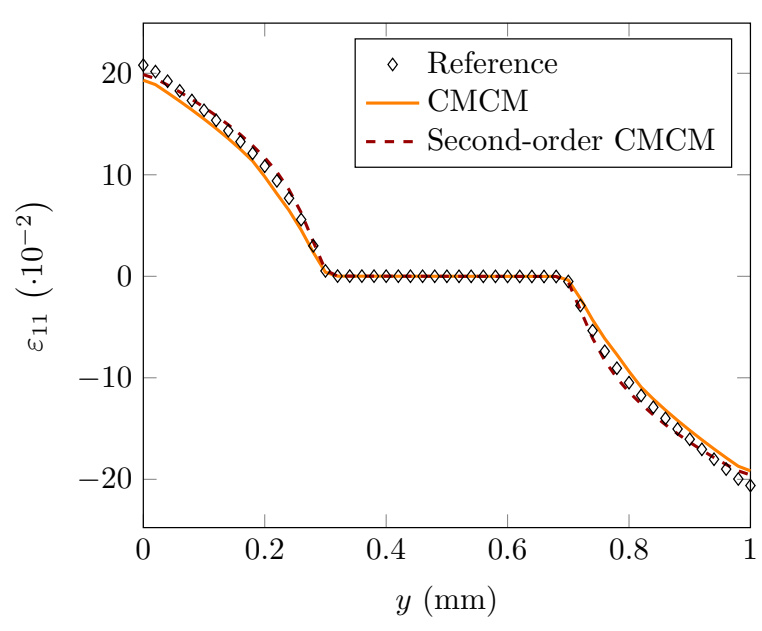

(a) Strain component $\varepsilon_{11}$

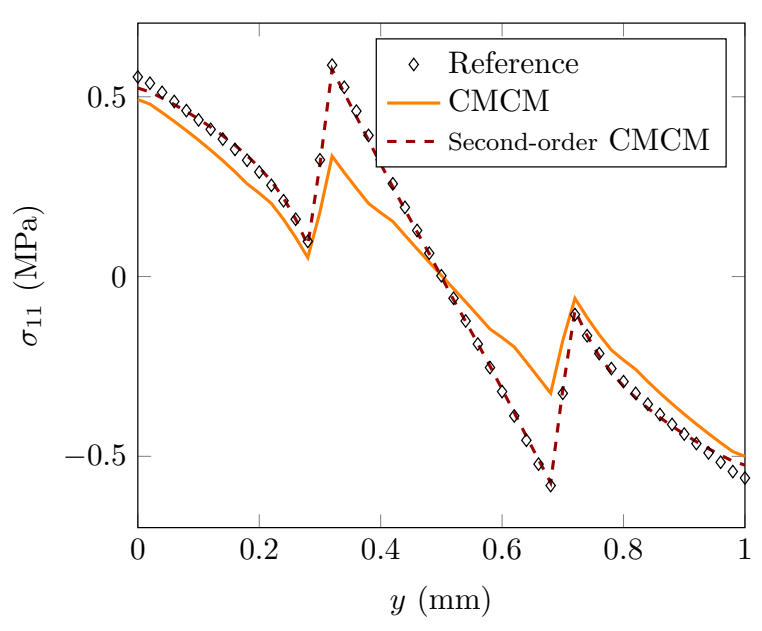

(b) Stress component $\sigma_{11}(\mathrm{MPa})$

Figure 24: Comparison of reference, CMCM, and Second-order CMCM solutions of local $(1,1)$ components long the line $(x=L / 2)$.

Here, the parameter $\beta$ (see section 3.2.1) was chosen to be zero. Different components of the local strain field $\boldsymbol{\varepsilon}(\mathbf{x})$ are compared with the reference solution, the CMCM and the Second-order CMCM in Figs. 21, 22, and 23. To better quantify the accuracy of each solution, the strain and stress $(1,1)$ components are plotted on the line $(x=L / 2)$ in Fig. 24 In these figures, only the most converged solutions, on coarse mesh 3, are displayed. In Fig. 25, the logarithmic error between the reference solution on a fine mesh and the reconstructed solution using $\mathrm{GFE}^{2}$ is shown. We can note that in this case, the Second-order CMCM gives a good convergence to the reference solution when refining the coarse mesh, while the CMCM does not converge.

In this example, the method with strain gradient leads to a more accurate solution at the same computational costs than the first-order method, as we recall that no additional degrees of freedom are introduced for that purpose.

In the next example, we consider a three dimensional problem to evaluate the capability of the method to handle a structure that is closer to practical applications. 

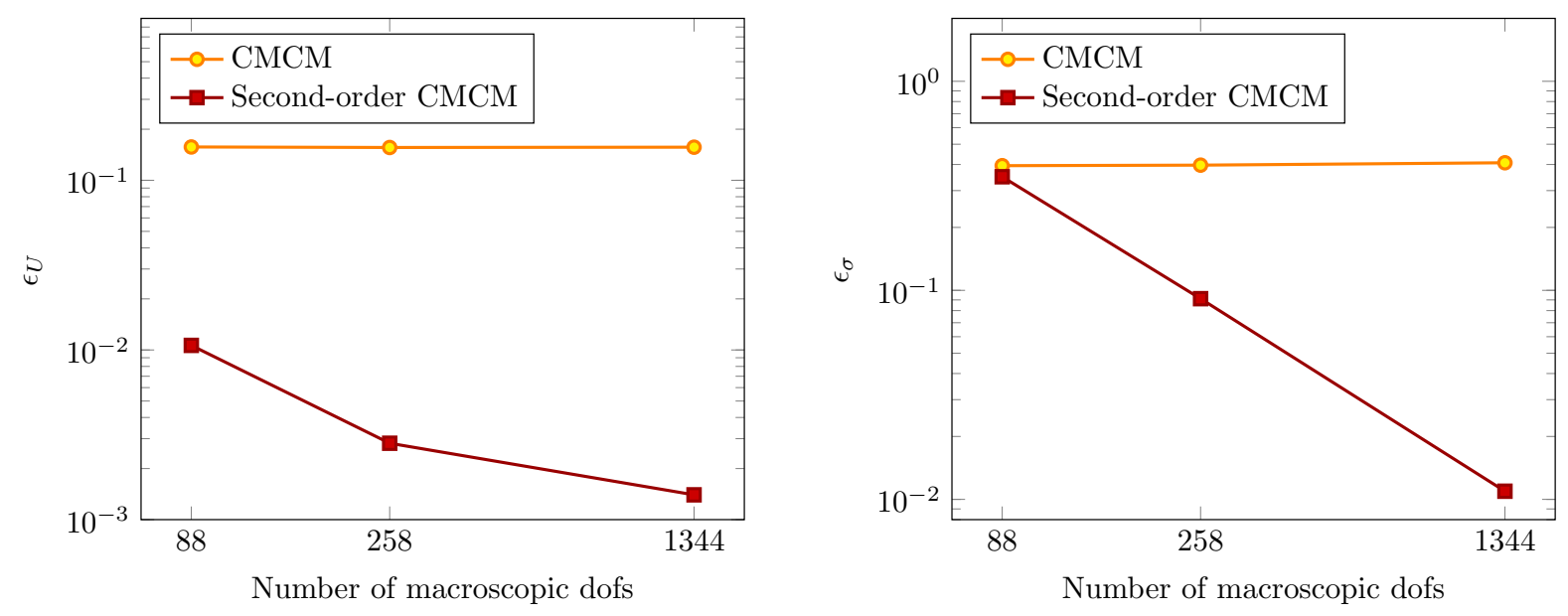

Figure 25: Minimum displacement (left) and maximum stress (right) using different coarse meshes.

\section{3. $3 D$ three point bending of a woven composite structure}

In this example, we show the capability of the method to handle industrial problems with a fine description of local heterogeneities within the structure. We consider the 3-point bending of a plain woven composite, as depicted in Fig. 26. The dimensions of the structure are $L=50 \mathrm{~mm}, b=10 \mathrm{~mm}$ and $h=1.1 \mathrm{~mm}$. As in the previous example, the loading is applied as pressure fields distributed along three lines $(x=3, z=0),(x=25, z=1.1)$ and $(x=47, z=0)$. The field distribution is described in Eq. 55 In order to remove rigid body motions, the nodal $z$-displacements on line $(x=0, z=0),(x=\mathrm{L}, z=0)$, the nodal $y$-displacements at nodes $(0,0,0),(\mathrm{L}, 0,0)$ and the nodal $x$-displacements at node $(0,0,0)$ are blocked.

In woven composites, there are three main scales of interest: the micro scale related to an explicit description of the fibers which compose the yarns, the meso scale related to the yarn's weaving, and the macro scale related to the whole structure. In this example, we consider a mesoscale description of the structure. Such simulations are usually avoided as not computationally tractable. However, we show that using CMCM, we are now able to solve the full problem including all the details of the macro and meso scales. In order to provide a reference solution, the problem is both solved using CMCM and the high performance parallel domain decomposition method AMPFETI (Adaptive Multi-Preconditioned Finite Element Tearing and Interconnect i.e. AMPFETI [7] which has been implemented in the finite element software Z-set at Safran Tech [8]. The solution obtained with the AMPFETI method is performed on a mesh involving $63 \times 10^{6}$ linear tetrahedral elements, corresponding to $37 \times 10^{6}$ dofs, while the CMCM computation involves a total of $37.1 \times 10^{6} \mathrm{dofs}$, distributed over 652 subdomains including $59.2 \times 10^{3}$ dofs (and $16.6 \times 10^{3}$ trilinear hexahedral elements). The discretizations of both methods are of comparable sizes. In CMCM, the global problem is computed on a coarse mesh involving only $16.6 \times 10^{3}$ trilinear hexahedral elements corresponding to $60.6 \times 10^{3}$ dofs. The mesh of a periodic subdomain at the meso scale is generated with the TexGen software [43], on a domain with dimensions $\left(2 \times 2 \times 0.22 \mathrm{~mm}^{3}\right)$, using linear tetrahedral elements. For the sake of simplicity, the parameter $\beta$ (see section 3.2.1 was chosen as zero. The parameters used to generate this representative unit cell are described in Table 4. For the comparison of the local fields, we re-localize one block column at the center of the structure (see Fig. 28) and compare the strain and stress fields within the red section.

\begin{tabular}{cccc}
\hline Yarn spacing $(\mathrm{mm})$ & Yarn width $(\mathrm{mm})$ & Fabric thickness $(\mathrm{mm})$ & Gap size \\
\hline 1 & 0.8 & $0.2(+10 \%$ option $)$ & 0 \\
\hline
\end{tabular}

Table 4: Parameters used in TexGen for the generation of the texture form. 


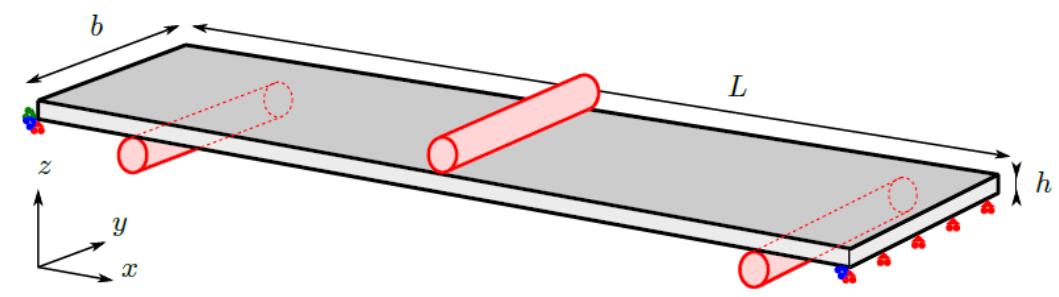

Figure 26: 3D woven composite beam structure problem: geometry and boundary conditions.

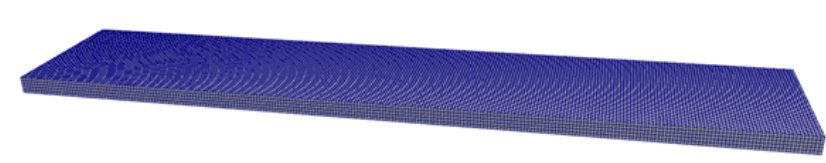

(a)

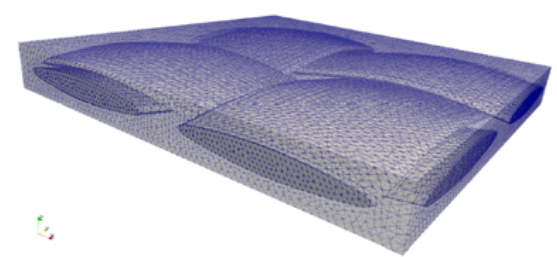

(b)

Figure 27: Finite element mesh of: (a) the macro structure and (b) one subdomain at the meso scale.

The behavior of the matrix is considered as elastic and isotropic (see Table 55). The yarns are considered as linear elastic orthotropic, and their properties are provided in Table 6.

\begin{tabular}{cc}
\hline Young's modulus $E(\mathrm{MPa})$ & Poisson's ratio $v$ \\
\hline 4000 & 0.3 \\
\hline
\end{tabular}

Table 5: Mechanical properties of the matrix.

\begin{tabular}{ccccccccc}
\hline $\mathrm{E}_{11}(\mathrm{MPa})$ & $\mathrm{E}_{22}(\mathrm{MPa})$ & $\mathrm{E}_{33}(\mathrm{MPa})$ & $v_{12}$ & $v_{23}$ & $v_{31}$ & $\mathrm{G}_{12}(\mathrm{MPa})$ & $\mathrm{G}_{23}(\mathrm{MPa})$ & $\mathrm{G}_{31}(\mathrm{MPa})$ \\
\hline 194400 & 8200 & 8200 & 0.3 & 0.3 & 0.0126 & 7000 & 3100 & 7000 \\
\hline
\end{tabular}

Table 6: Mechanical properties of the yarns

The constitutive relation of the stress and strain in the yarns is given in matrix form as:

$$
\left[\begin{array}{l}
\sigma_{11} \\
\sigma_{22} \\
\sigma_{33} \\
\sigma_{12} \\
\sigma_{13} \\
\sigma_{23}
\end{array}\right]=\left[\begin{array}{cccccc}
1 / \mathrm{E}_{11} & -v_{12} / \mathrm{E}_{22} & -v_{13} / \mathrm{E}_{33} & 0 & 0 & 0 \\
-v_{12} / \mathrm{E}_{11} & 1 / \mathrm{E}_{22} & -v_{23} / \mathrm{E}_{33} & 0 & 0 & 0 \\
-v_{13} / \mathrm{E}_{11} & -v_{23} / \mathrm{E}_{22} & 1 / \mathrm{E}_{33} & 0 & 0 & 0 \\
0 & 0 & 0 & 1 / \mathrm{G}_{12} & 0 & 0 \\
0 & 0 & 0 & 0 & 1 / \mathrm{G}_{13} & 0 \\
0 & 0 & 0 & 0 & 0 & 1 / \mathrm{G}_{23}
\end{array}\right]^{-1}\left[\begin{array}{l}
\varepsilon_{11} \\
\varepsilon_{22} \\
\varepsilon_{33} \\
2 \varepsilon_{12} \\
2 \varepsilon_{13} \\
2 \varepsilon_{23}
\end{array}\right] .
$$

These orthotropic properties are defined in a frame attached to the material orientation of the yarns. In each main (warp and weft) yarn direction, the above tensor is further slightly rotated according to their normal orientation, which varies due to weaving form. However, in this example we simplify the problem by assuming that each yarn only has one constant direction. To be specific, for the warp yarn elements (along $x$ ), the above tensor is unchanged, but for the weft yarn elements (along $y$ ), it is rotated $90^{\circ}$. 


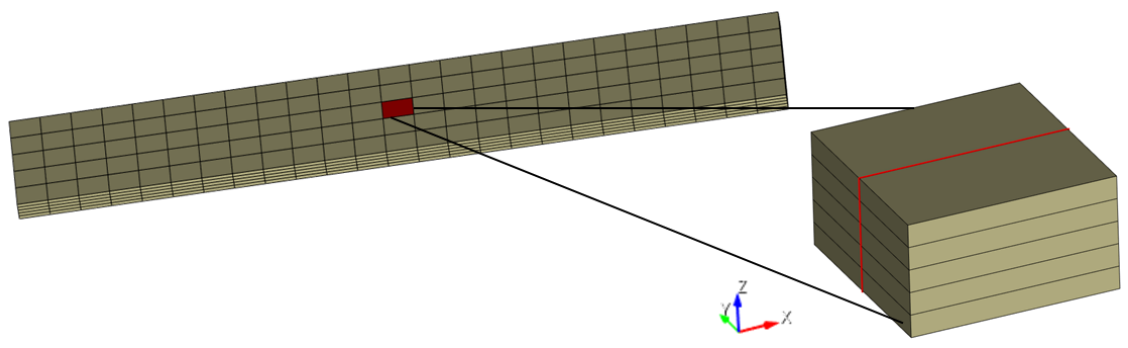

Figure 28: Illustration of the section used for result comparison.

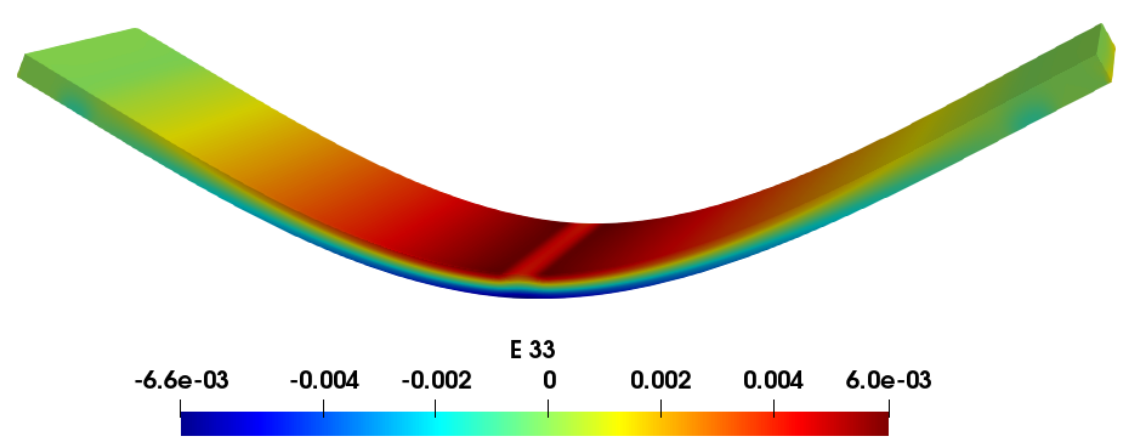

Figure 29: Macro strain solution $\bar{\varepsilon}_{33}(\mathbf{x})$ on the deformed coarse mesh using the Second-order CMCM.

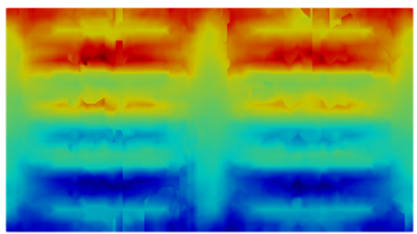

(a) Reference (AMPFETI)

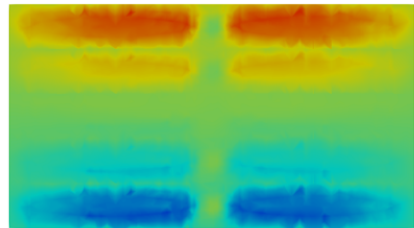

(b) $\mathrm{CMCM}$

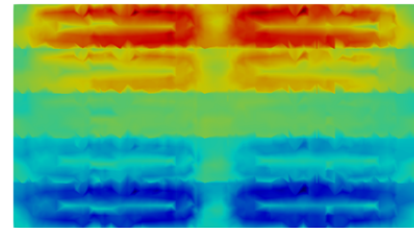

(c) Second-order CMCM

$$
\begin{aligned}
& \varepsilon_{22}(\mathbf{x})
\end{aligned}
$$

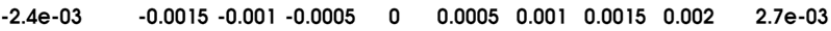

Figure 30: Relocalized strain solution $\varepsilon_{22}(\mathbf{x})$ : (a) reference solution (AMPFETI); (b) basic-CMCM; (c) Second-order CMCM.

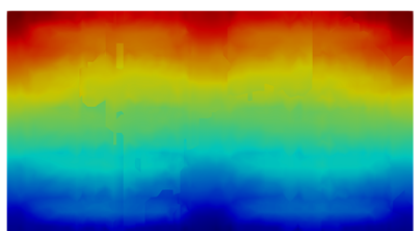

(a) Reference (AMPFETI)

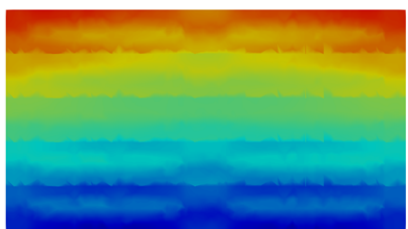

(b) $\mathrm{CMCM}$

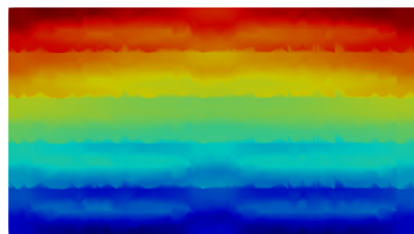

(c) Second-order CMCM

$$
\varepsilon_{33}(\mathbf{x})
$$

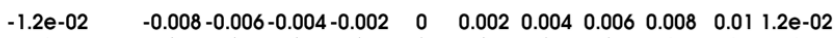

Figure 31: Relocalized strain solution $\varepsilon_{33}(\mathbf{x})$ : (a) reference solution (AMPFETI); (b) basic-CMCM; (c) Second-order CMCM. 


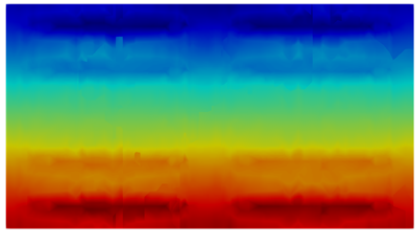

(a) Reference (AMPFETI)

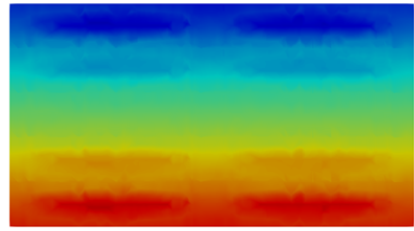

(b) $\mathrm{CMCM}$

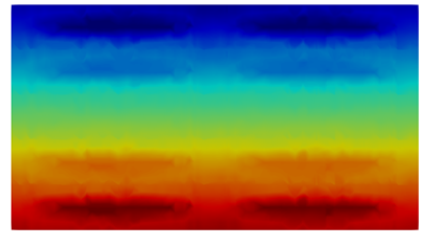

(c) Second-order CMCM

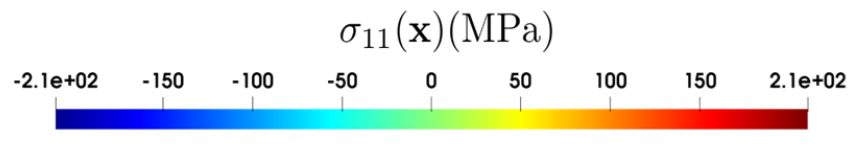

Figure 32: Relocalized stress solution $\sigma_{11}(\mathbf{x})(\mathrm{MPa})$ : (a) reference solution (AMPFETI); (b) basic-CMCM; (c) Second-order CMCM.

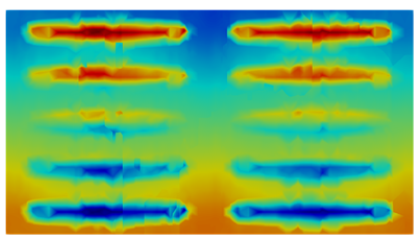

(a) Reference (AMPFETI)

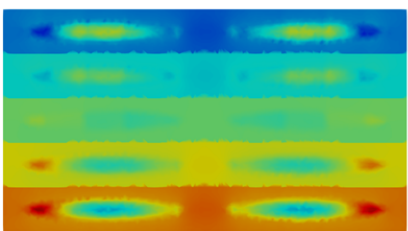

(b) $\mathrm{CMCM}$

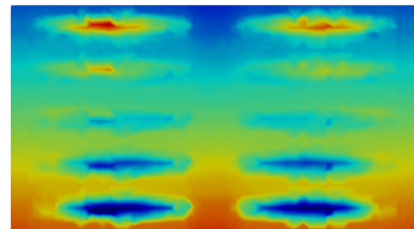

(c) Second-order CMCM

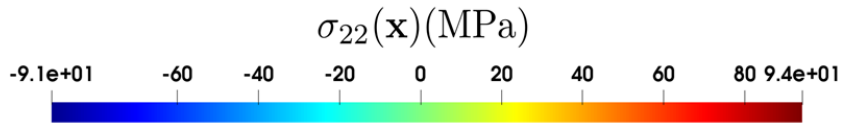

Figure 33: Relocalized stress solution $\sigma_{22}(\mathbf{x})(\mathrm{MPa})$ : (a) reference solution (AMPFETI); (b) basic-CMCM; (c) Second-order CMCM.

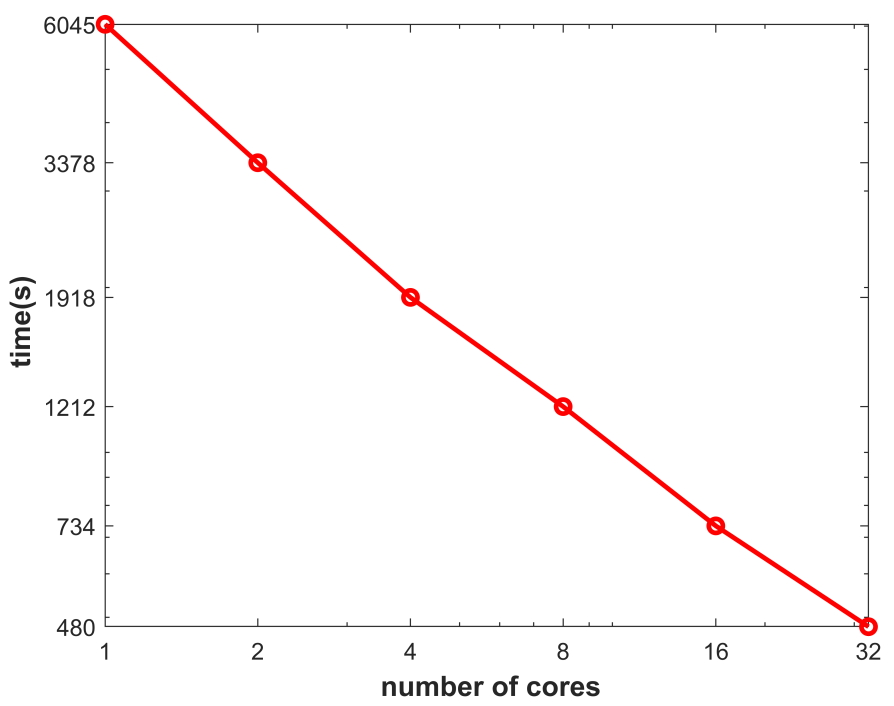

Figure 34: Computational time in function of number of cores.

Fig. 34 shows the computational time of the local and global problem for different number of processors. It is worth noting that all local problems are independent, and thus can all be performed in parallel. We can then observe that the computational times are decreased linearly with the number of available cores 
We can clearly see on the different results that the agreement between AMPFETI and CMCM is satisfying, and that the use of the gradient enhancement improves the agreement with the reference solution. For comparison, the AMPFETI calculation took 10 minutes on 744 cores. The CMCM took 73 minutes in total (including parallel calculations and solving the problem on the coarse mesh), but on a standard 24 cores workstation.

\subsection{Large scale simulation involving 1.3 Billion of dof}

In this example, we demonstrate the capabilities of the method to handle fully detailed structures with large dimensions, and a larger scale ratio between structure dimensions and microstructural details, as found in some industrial applications. The structure has the same characteristics as the one presented in the previous example, but involves $49 \times 10 \times 10$ subdomains (see Fig. 35) as defined in Fig 27(b). The material properties and the boundary conditions are the same as in the previous section. The complete model involves roughly 1.3 billion dofs. It is obvious than in such situation, most available FEM solvers are not able to handle such a large simulation even with high performance computing. The coarse mesh involves 96,800 trilinear hexahedral elements corresponding to 321,489 dofs (Figure 37), indicating that there is still room for possible refinement of this mesh to improve accuracy. Here again, the parameter $\beta$ (see section 3.2.1) was chosen as zero. To provide a more accurate solution in the vicinity of the load, the coarse mesh is refined in some regions near the pressure fields, as depicted in Fig. 37.a).

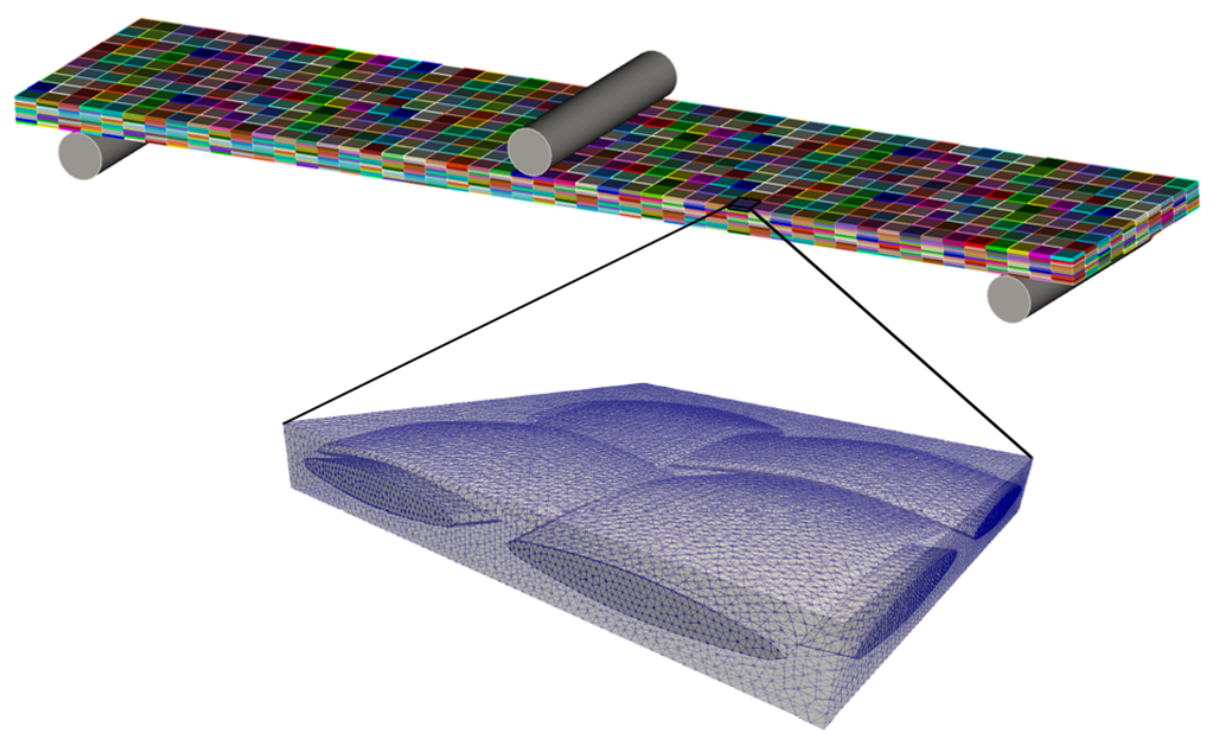

Figure 35: Structure decomposed into $49 \times 10 \times 10$ subdomains and involvin $1.3 \times 10^{9}$ dofs.

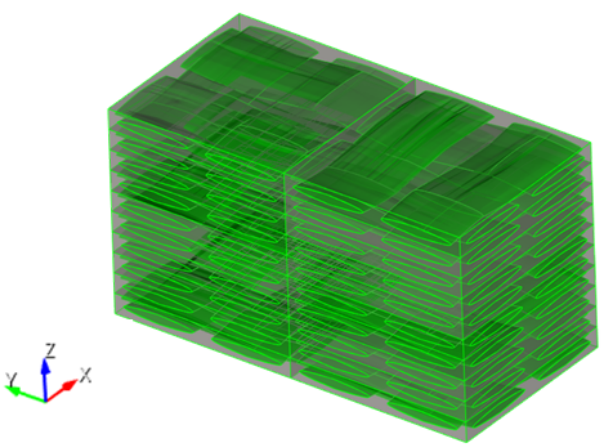

Figure 36: Subdomains chosen for relocalization of strain and stress fields. 
For illustration, we relocalize the local strain and stress fields in two block columns of the structure (see Fig. 36. As we have already shown that, for such bending-dominated cases, the Second-order CMCM method improves the accuracy without additional costs, we have here only used the Second-order CMCM method.

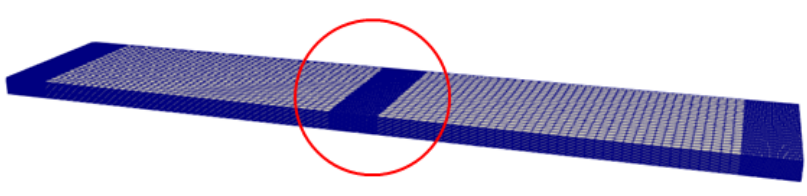

(a) Considered structure

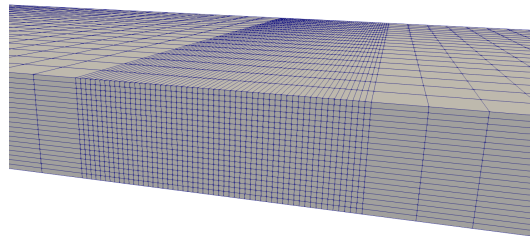

(b) Zoom-in at the center

Figure 37: Coarse mesh: (a) global view and (b) zoom-in at the center of the structure.

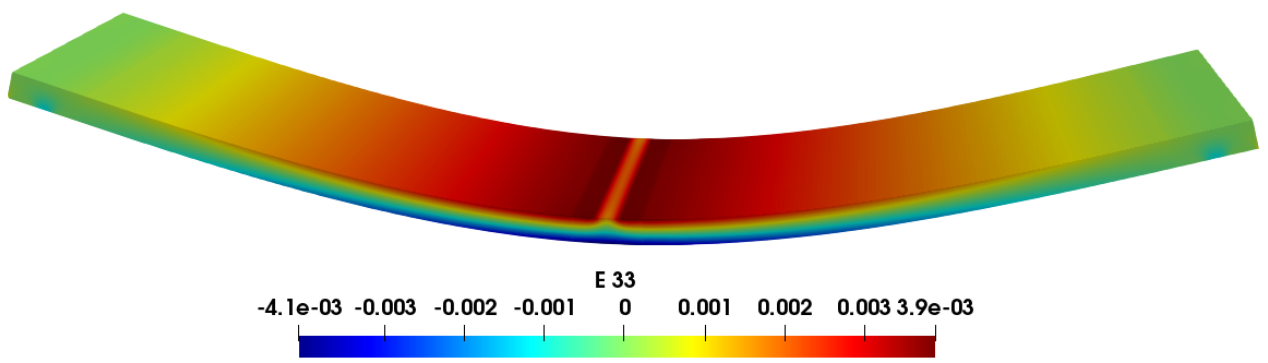

Figure 38: Macro strain solution $\bar{\varepsilon}_{33}(\mathbf{x})$ on the deformed coarse mesh.
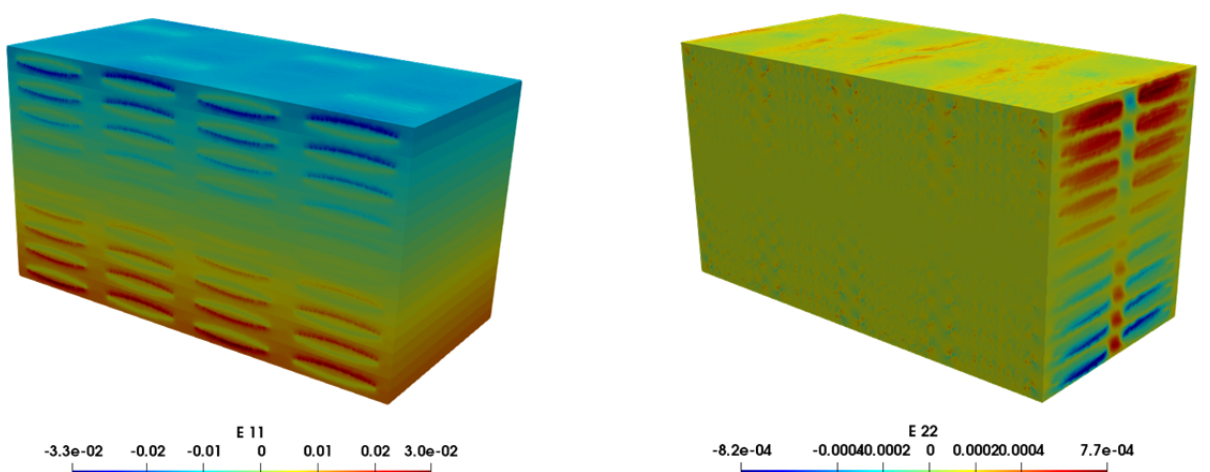

Figure 39: Relocalized solution: $\varepsilon_{11}(\mathbf{x})$ (left) and $\varepsilon_{22}(\mathbf{x})$ (right).

The total simulation time was 20 minutes for each subdomain off-line calculation (performed in parallel) and the coarse mesh calculation took roughly 5 days, on 32 cores. Then, the present method has a very high potential to study fully detailed composite structures without any assumptions on scale separation and periodicity. 

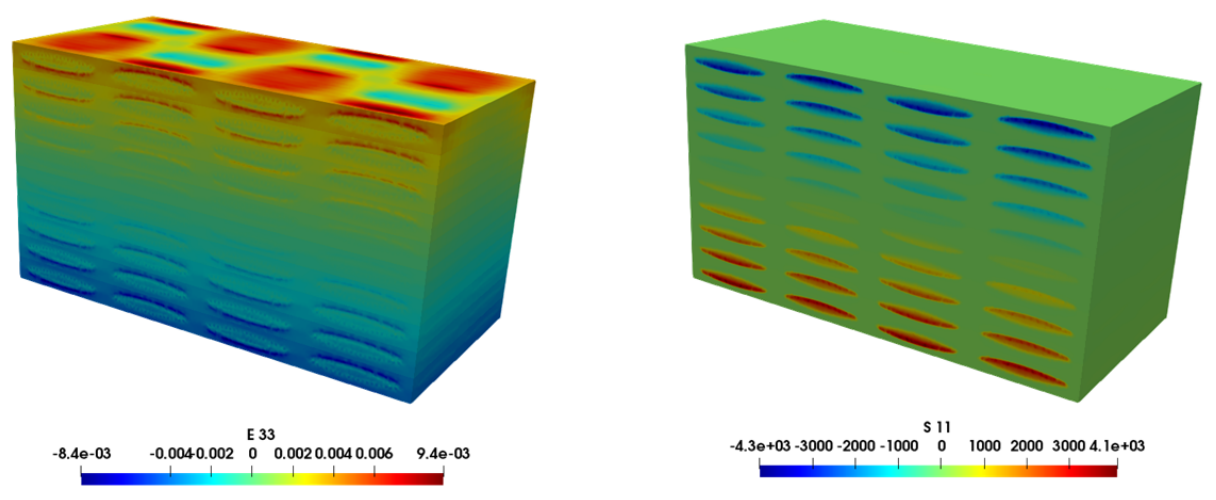

Figure 40: Relocalized solution: $\varepsilon_{33}(\mathbf{x})$ (left) and $\sigma_{11}(\mathbf{x})(\mathrm{MPa})$ (right).
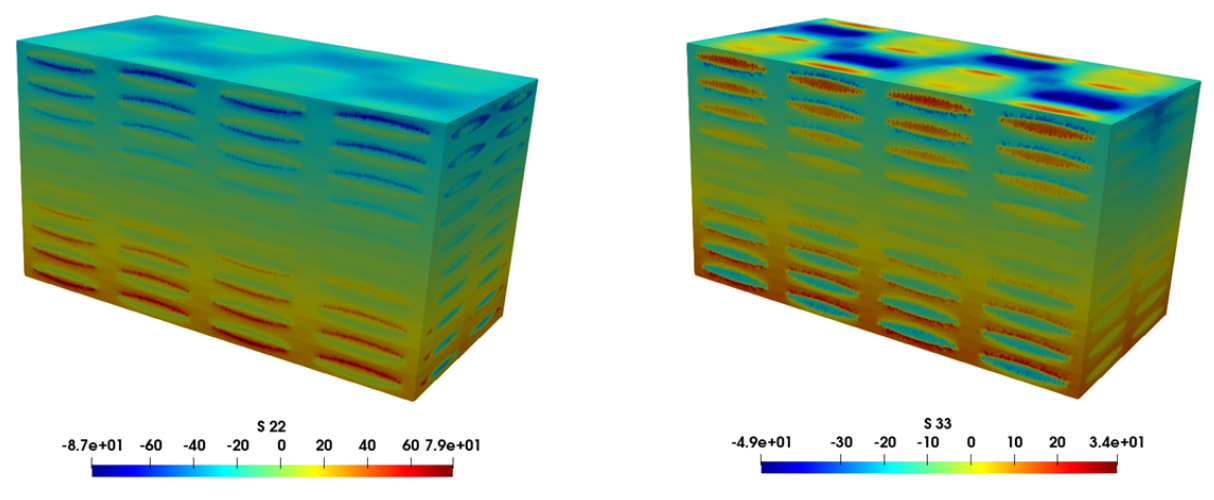

Figure 41: Relocalized solution: $\sigma_{22}(\mathbf{x})(\mathrm{MPa})$ (left) and $\sigma_{33}(\mathbf{x})(\mathrm{MPa})$ (right).

\section{Conclusions}

In this work, we have introduced a new method for parallel computations of large heterogeneous structures, called Coarse Mesh Condensation Multiscale Method (CMCM). In this technique, the key idea is to construct an approximation of the full field solution in the structure at the scale of heterogeneities by combining solutions computed on a fine mesh on subdomains decomposing the structure and a solution computed on a coarse mesh. By analogy with the $\mathrm{FE}^{2}$ method [24], elements of the coarse mesh provide information for applying Dirichlet on the boundaries of subdomains covering the structure. As a result, the full-field solution is approximated over the whole structure at the cost of a finite number of calculations on subdomains which can be solved in parallel and a coarse mesh problem with a low number of dofs. In this paper, we have only treated the linear case. In this situation, matrix relationships can be obtain to relate the dofs of the coarse mesh and the dofs of the fine mesh subdomains solutions, resulting in a one-iteration scheme only. An extension of the approach to strain gradient has been proposed to enhance the solution in case of global bending of the structures. The accuracy of the method has been tested through benchmark problems involving subdomains crossing the interfaces in the case of high contrast between the phase, which is known to constitute a difficult case for iterative domain decomposition methods. Finally, we have presented an industrial-sized example of a composite beam involving 1.3 billion dofs which has been computed on a standard 32-core standard workstation to show the potential of the method. 


\section{Acknowledgements}

The authors would like to thank Professor Tarek Zohdi at University of California, Berkeley, for his comments and suggestions concerning an early preprint of this manuscript. They are also grateful to F. Feyel, C. Rey and T. Rose for their help in proofreading and improving this article.

\section{References}

[1] C. Farhat, F.-X. Roux, A method of finite element tearing and interconnecting and its parallel solution algorithm, International Journal for Numerical Methods in Engineering 32 (6) (1991) 1205-1227.

[2] P. Le Tallec, Y.-H. De Roeck, M. Vidrascu, Domain decomposition methods for large linearly elliptic three-dimensional problems, Journal of Computational and Applied Mathematics 34 (1) (1991) 93-117.

[3] D. J. Rixen, C. Farhat, A simple and efficient extension of a class of substructure based preconditioners to heterogeneous structural mechanics problems, International Journal for Numerical Methods in Engineering 44 (4) (1999) 489-516.

[4] P. Gosselet, C. Rey, D. J. Rixen, On the initial estimate of interface forces in FETI methods, Computer Methods in Applied Mechanics and Engineering 192 (25) (2003) 2749-2764.

[5] N. Spillane, V. Dolean, P. Hauret, F. Nataf, D. Rixen, Solving generalized eigenvalue problems on the interfaces to build a robust two level FETI method, International Journal for Numerical Methods in Engineering (2012) (2012).

[6] P. Gosselet, D. Rixen, F.-X. Roux, N. Spillane, Simultaneous FETI and block FETI: Robust domain decomposition with multiple search directions, International Journal for Numerical Methods in Engineering 104 (10) (2015) 905-927.

[7] N. Spillane, An adaptive multipreconditioned conjugate gradient algorithm, SIAM Journal on Scientific Computing 38 (3) (2016) A1896A1918.

[8] C. Bovet, A. Parret-Fréaud, N. Spillane, P. Gosselet, Adaptive multipreconditioned FETI: scalability results and robustness assessment, Computers \& Structures 193 (2017) 1-20.

[9] P. Ladevèze, O. Loiseau, D. Dureisseix, A micro-macro and parallel computational strategy for highly heterogeneous structures, International Journal for Numerical Methods in Engineering 52 (1-2) (2001) 121-138.

[10] P. Ladevèze, J.-C. Passieux, D. Néron, The latin multiscale computational method and the proper generalized decomposition, Computer Methods in Applied Mechanics and Engineering 199 (21-22) (2010) 1287-1296.

[11] K. Stüben, Algebraic multigrid (AMG): experiences and comparisons, Applied mathematics and computation 13 (3-4) (1983) 419-451.

[12] J. Ruge, K. Stüben, Efficient solution of finite difference and finite element equations by algebraic multigrid AMG, Gesellschaft f. Mathematik u. Datenverarbeitung, 1984.

[13] J. W. Ruge, K. Stüben, Algebraic multigrid, in: Multigrid methods, SIAM, 1987, pp. 73-130.

[14] K. Stüben, A review of algebraic multigrid, in: Numerical Analysis: Historical Developments in the 20th Century, Elsevier, 2001, pp. $331-359$.

[15] T. I. Zohdi, P. Wriggers, A domain decomposition method for bodies with heterogeneous microstructure basedon material regularization, International Journal of Solids and Structures 36 (17) (1999) 2507-2525.

[16] T. I. Zohdi, P. Wriggers, C. Huet, A method of substructuring large-scale computational micromechanical problems, Computer Methods in Applied Mechanics and Engineering 190 (43-44) (2001) 5639-5656.

[17] M. Hautefeuille, J.-B. Colliat, A. Ibrahimbegovic, H. Matthies, P. Villon, A multi-scale approach to model localized failure with softening, Computers \& Structures 94 (2012) 83-95.

[18] A. Huerta, E. Nadal, F. Chinesta, Proper generalized decomposition solutions within a domain decomposition strategy, International Journal for Numerical Methods in Engineering 113 (13) (2018) 1972-1994.

[19] E. Weinan, B. Engquist, X. Li, W. Ren, E. Vanden-Eijnden, Heterogeneous multiscale methods: a review, Commun. Comput. Phys 2 (3) (2007) 367-450.

[20] A. Abdulle, E. Weinan, B. Engquist, E. Vanden-Eijnden, The heterogeneous multiscale method, Acta Numerica 21 (2012) 1-87.

[21] T. Y. Hou, X.-H. Wu, A multiscale finite element method for elliptic problems in composite materials and porous media, Journal of computational physics 134 (1) (1997) 169-189.

[22] Y. Efendiev, T. Y. Hou, Multiscale finite element methods: theory and applications, Vol. 4, Springer Science \& Business Media, 2009.

[23] T. I. Zohdi, P. Wriggers, An Introduction to Computational Micromechanics: Corrected Second Printing, 1st Edition, Lecture Notes in Applied and Computational Mechanics 20, Springer-Verlag Berlin Heidelberg, 2005.

[24] F. Feyel, A multilevel finite element method $\left(\mathrm{FE}^{2}\right)$ to describe the response of highly non-linear structures using generalized continua, Computer Methods in Applied Mechanics and Engineering 192 (28-30) (2003) 3233-3244.

[25] S. Forest, K. Sab, Cosserat overall modelling of heterogeneous materials, Mechanics Research Communications 25(4) (1998) 449-454.

[26] V. Kouznetsova, M. Geers, W. Brekelmans, Multi-scale constitutive modeling of heterogeneous materials with gradient enhanced computational homogenization scheme, International Journal for Numerical Methods in Engineering 54 (2002) 1235-1260.

[27] V. Kouznetsova, M. Geers, W. Brekelmans, Multi-scale second-order computational homogenization of multi-phase materials: a nested finite element solution strategy, Computer Methods in Applied Mechanics and Engineering (2003).

[28] X. Yuan, Y. Tomita, A micromechanical approach of nonlocal modeling for media with periodic microstructures, Mechanics Reasearch Communications 35 (2008) 126133

[29] F. Bouyge, I. Jasiuk, M. Ostoja-Starzewski, A micromechanically based couple-stress model of an elastic two-phase composite, International Journal of Solids and Structures 38 (10-13) (2001) 1721-1735.

[30] T.-H. Tran, V. Monchiet, G. Bonnet, A micromechanics-based approach for the derivation of constitutive elastic coefficients of strain-gradient media, International Journal of Solids and Structures 49 (2012) 783-792. 
[31] A. Tognevi, M. Guerich, J. Yvonnet, A multi-scale modeling method for heterogeneous structures without scale separation using filter-based homogenization scheme, International Journal for Numerical Methods in Engineering (2016).

[32] T. Hui, C. Oskay, A nonlocal homogenization model for wave dispersion in dissipative composite materials, International Journal of Solids and Structures 50 (2013) 38-48.

[33] F. Feyel, Multiscale $\mathrm{FE}^{2}$ elastoviscoplastic analysis of composite structure, Computational Material Science 16(1-4) (1999) $433-454$.

[34] F. Feyel, J.-L. Chaboche, $\mathrm{FE}^{2}$ multiscale approach for modelling the elastoviscoplastic behaviour of long fibre SiC/Ti composite materials, Computer Methods in Applied Mechanics and Engineering 183 (3-4) (2000) 309-330.

[35] J. Yvonnet, Q.-C. He, The reduced model multiscale method (R3M) for the non-linear homogenization of hyperelastic media at finite strains, Journal of Computational Physics 223 (2007) 341-368.

[36] I. Ozdemir, W. Brekelmans, M. Geers, Computational homogenization for heat conduction in heterogeneous solids, International Journal for Numerical Methods in Engineering 73 (2) (2008) 185-204.

[37] M. Geers, V. Kouznetsova, W. Brekelmans, Multi-scale computational homogenization: Trends and challenges, Journal of Computational and Applied Mathematics 234 (7) (2010) 2175-2182.

[38] E. Coenen, V. Kouznetsova, E. Bosco, M. Geers, A multi-scale approach to bridge microscale damage and macroscale failure: a nested computational homogenization-localization framework, International journal of fracture 178 (1-2) (2012) $157-178$.

[39] L. Xia, P. Breitkopf, Concurrent topology optimization design of material and structure within fe 2 nonlinear multiscale analysis framework, Computer Methods in Applied Mechanics and Engineering 278 (2014) 524-542.

[40] R. Mindlin, N. Eshel, On first strain-gradient theories in linear elasticity, International Journal of Solids and Structures 4 (1) (1968) $109-124$.

[41] N. Auffray, J. Dirrenberger, G. Rosi, A complete description of bi-dimensional anisotropic strain-gradient elasticity, International Journal of Solids and Structures 69 (2015) 195-206.

[42] J. Yvonnet, N. Auffray, V. Monchiet, Computational second-order homogenization of materials with effective anisotropic strain gradient behavior, Submitted.

[43] H. Lin, L. P. Brown, A. C. Long, Modelling and simulating textile structures using texgen, in: Advanced Materials Research, Vol. 331, Trans Tech Publ, 2011, pp. 44-47.

[44] P. Gosselet, C. Rey, Non-overlapping domain decomposition methods in structural mechanics, Archives of Computational Methods in Engineering 13 (4) (2006) 515. 